\title{
Scenario Generation for Electric Vehicles' \\ Uncertain Behavior in a Smart City Environment
}

Author: João Soares (Corresponding author)

Affiliation: GECAD - Research Group on Intelligent Engineering and Computing for Advanced Innovation and Development - Polytechnic of Porto (IPP)

Address: R. Dr. António Bernardino de Almeida, 431, 4200-072 Porto, Portugal

Email: joaps@isep.ipp.pt

Author: Nuno Borges

Affiliation: GECAD - Research Group on Intelligent Engineering and Computing for Advanced Innovation and Development - Polytechnic of Porto (IPP)

Address: R. Dr. António Bernardino de Almeida, 431, 4200-072 Porto, Portugal

Email: ndsbs@isep.ipp.pt

Author: Mohammad Ali Fotouhi Ghazvini,

Affiliation: GECAD - Research Group on Intelligent Engineering and Computing for Advanced Innovation and Development - Polytechnic of Porto (IPP)

Address: R. Dr. António Bernardino de Almeida, 431, 4200-072 Porto, Portugal

Email: ma.fotouhi@gmail.com

Author: Zita Vale

Affiliation: GECAD - Research Group on Intelligent Engineering and Computing for Advanced Innovation and Development - Polytechnic of Porto (IPP)

Address: R. Dr. António Bernardino de Almeida, 431, 4200-072 Porto, Portugal

Email: zav@isep.ipp.pt

Author: P. B. de Moura Oliveira

Affiliation: INESC TEC - INESC Technology and Science - UTAD University

Address: Quinta de Prados, 5000-801 Vila Real, Portugal

Email: oliveira@utad.pt 


\title{
Scenario Generation for Electric Vehicles' Uncertain Behavior in a Smart City Environment
}

João Soares ${ }^{1,}{ }^{*}$, Nuno Borges ${ }^{1}$, Mohammad Ali Fotouhi Ghazvini ${ }^{1}$, Zita Vale ${ }^{1}$, P. B. de

\author{
Moura Oliveira ${ }^{2}$
}

${ }_{1}^{1}$ GECAD - Research Group on Intelligent Engineering and Computing for Advanced Innovation and Development - Polytechnic of Porto (IPP) R. Dr. António Bernardino de Almeida, 431, 4200-072 Porto, Portugal 2 INESC TEC - INESC Technology and Science - UTAD University Quinta de Prados, 5000-801 Vila Real, Portugal

*Corresponding author: João Soares

Tel.: +35122 8340500; Fax: +35122 8321159, joaps@isep.ipp.pt

\section{ABSTRACT}

This paper presents a framework and methods to estimate electric vehicles' possible states, regarding their demand, location and grid connection periods. The proposed methods use the Monte Carlo simulation to estimate the probability of occurrence for each state and a fuzzy logic probabilistic approach to characterize the uncertainty of electric vehicles' demand. Day-ahead and hour-ahead methodologies are proposed to support the smart grids' operational decisions. A numerical example is presented using an electric vehicles fleet in a smart city environment to obtain each electric vehicle possible states regarding their grid location.

KEYWORDS: Big Data; Electric Vehicles; Fuzzy Logic; Monte Carlo Simulation; Smart City. 


\section{Introduction}

Electric Vehicles (EVs) are one of the main proposed alternatives to replace the conventional vehicles powered by fossil fuels [1]. Despite the first EVs being developed in the $19^{\text {th }}$ century, more than 100 years ago, petrol vehicles have overcome them due to their higher autonomy and low fuel prices [2]. Currently, the situation is changing and important advances have been achieved in the batteries' technology, i.e., the energy density has increased by several-folds [3]. Successful EVs have been settling on the market, e.g., Nissan Leaf, Tesla S, and Renault Zoe, together sold more than 200,000 vehicles worldwide.

The EVs' batteries charging is possible by using both regenerative braking and by connection of the electrical outlet. However, the last is by far the main source of EV's energy [4]. This means that EVs are highly dependent to the electricity grid, which may affect the power system operation [5]. To mitigate to those effects, it is important that both transmission and distribution grid system are integrated and smart according to [6]. In [7], the relation of electric mobility between transportation and the power grid is analyzed by providing the EVs' daily patterns at different grid locations, while showing that control can be used to mitigate the network congestion and to change the transport behavior. A possibility widely studied but not yet completely developed as a commercial plan is using EVs not only as a load, but also as a source to the grid. This concept is often designated as Vehicle-to-Grid (V2G) [8]. Moreover, EVs could be used to provide energy to the buildings as proposed in [9], namely for the interactions of EVs with a medium office building. The economic viability of the V2G technology and the relevant business models have already been addressed in [10-12]. Indeed, V2G can contribute to increase stability, reliability, serve as storage and lower electricity costs, e.g. by reducing the $\$ 12$ billion per year in USA related with balancing [10]. In [11, a 
research is conducted to identify actors and operational activities affected by EVs integration, while proposing solutions for short term planning and operational tasks including load management and V2G. A regulatory framework and business models for network operators and EVs aggregators are described in [11] regarding opportunities for V2G applications, such as peak power and frequency regulation. Models regarding EVs' bidding and optimal charging strategies are proposed in [13]. The problem of optimally managing a microgrid considering V2G interactions is addressed in [14-16]. In [14], a robust optimization is proposed to tackle uncertainty of vehicles' behavior in the energy management problem considering V2G services. In [15], the V2G frequency regulation service under an energy aggregator concept is investigated using a optimization approach. In [16], a day-ahead energy scheduling model is proposed to address the use of distributed energy resources, including massive V2G deployment.

Integration of renewable generation and EVs will affect modernized power systems' planning and operation, mainly due to uncertainties in this paradigm. Information regarding EVs' location and expected demand would ultimately lead to a better grid planning and operation by reducing the degree of uncertainties. One EV user can leave home early in the morning at 8:00 and return from work at 18:00. This behavior may be similar in working days. However, some deviations and rare events can occur such as a late leave in the morning, e.g. at 9:00. Efficiently managing these deviations is important to improve future smart grids' management under the smart cities' concept. Smart city is defined in [17] as a city in which Information and Communication Technology (ICT) is merged with traditional infrastructures, coordinated and integrated using the latest digital technologies. This type of cities represents a community interconnected and sustainable, comfortable, attractive and secure. These Smart Cities can be integrated in a solution considering electricity, water and 
gas consumptions, as well as heating and cooling systems, public safety, wastes management and mobility. In the context of smart cities and due to ICT improvements, big data will be available for smart grid operators [18]. Real-time records regarding EVs' location, time of charge and energy charged, could be maintained in appropriate data storage systems, e.g. a computer cloud. This data can be processed to be useful for operators in a later stage.

\subsection{Literature review}

Several methodologies have been proposed in the literature to deal with the uncertainty of EVs in a power grid. The MCS method is employed in [19-22] to deal with the uncertainties that are associated with the energy planning of power systems, buildings and microgrids. A multi-criterion optimization method for renewable energy system design towards net zero energy building is proposed in [19], considering the uncertainties of energy generation and consumption. The MCS is conducted to estimate the power mismatch and annual energy balance. In [20], Monte Carlo analysis is used to analyze the impacts of more aggressive U.S. residential and commercial building-energy codes and equipment standards on energy consumption and energy service costs. Authors claim that the study is robust to significant uncertainties by using MCS. In [21], a MCS is used to generate a set of random inputs, which are uncertain variables, such as: learning effect, fuel price and $\mathrm{CO}_{2} \cos$ in the optimization of Korean energy planning for a sustainable future. In [22], authors use the Sequential MCS to generate the net load of a microgrid, for two different scenarios, one without considering the stochastic correlations and the other considering stochastic

correlations. The EVs and the uncertainties associated with their operation are not considered in [19-22]. 
In [1], authors study the impact on power system reliability considering uncertainty in the EVs. The work proposes to use Monte Carlo Simulation (MCS) to generate a set of 10,000 scenarios and then K-means clustering to reduce the complexity to 3 scenarios. The work does not consider any historical database but rather a presumable driving pattern for a representative fleet of EVs. Moreover, the location of EVs is ignored for their purpose. In [23] a Markov Chain Monte Carlo method is employed to model the household occupancy based on time use surveys, which together with weather variables, neighborhood characteristics and behavioral data, are used to model the switching pattern of appliances. Authors claim that EVs are considered in the model to estimate the residential electrical load. In [24], a PEV aggregator model is used. PEV aggregators submit three forecasted amounts for the total available capacity, required state of charge, and the available power for one hour in an EV. MCS is used to obtain different samples from the distributions of the uncertain variables. The estimated variables are capacity of EVs, state of charge and available power in an aggregated way. In [25] a MCS is used to build up a distribution of possible demands on trial networks for a single peak load day to evaluate distribution network impacts. The MCS is evaluated by individual user and the data for further analysis are obtained by combining the domestic load profile and EV charging profiles. In [26], a stochastic model is proposed to enable the optimal charging of EVs in the presence of renewable energy sources. The EVs are not considered individually but are clustered into fleets. Only the energy needs of the fleet are considered. In fact, the MCS method is the same as published in [1], but modified for a different purpose. The work reported in [27] uses MCS to obtain EVs' profiles regarding their locations with the aim to evaluate the impacts on the distribution networks. Scenarios with $25 \%$ and $50 \%$ of EVs' penetration are tested in a small island of Azores in Portugal. A model to estimate a fleet of plug-in electric vehicles energy demand is proposed 
in [28]. This model is used to evaluate the impacts of charging in distribution networks. This work uses MCS to generate vehicles' trips according to users' behavior, types of vehicles, periods of charging and the temperature effect. In [29] an EV demand model for load flow studies is developed. The model considers the EVs' demand as PQ buses with stochastic characteristics using queueing theory as a function of the charging time. This method was tested considering a case study with only one type of vehicle and does not take into account the changing behavior along the day. The same method has been applied in [30], to develop a probabilistic constrained load flow with the presence of EVs. In [31] a fuzzy logic (FL) control strategy is proposed for an EV energy management system with dual source power (battery and super capacitor). This architecture is proposed in order to satisfy EVs energy requirements, improving both the EVs efficiency as well as the overall performance of the system.

\subsection{Contributions}

This paper proposes a way to estimate the EVs' possible states (set of scenarios) regarding their location, grid connection periods and the energy demand. Predicting EVs' events is helpful and valuable for smart grids' management, due to their stochastic nature. In this context, an accurate set of stochastic scenarios can help operators to reduce their operation costs [32]. Therefore, reliable tools are very important to obtain these scenarios. For instance, they can help in designing adequate demand response programs, to offer different tariffs and location-based tariffs, to improve the use of $\mathrm{V} 2 \mathrm{G}$ technology, and to reduce network problems, such as congestion.

The research reported in this paper envisages a cloud-based operation with dispersed sensing devices, namely in the context of Internet of Things (IoT) and big data. It is assumed 
that each EV has appropriate sensing devices to collect and generate data. The data can be stored locally in the EV or accessed by cloud applications and stored remotely (see Fig. 1). In the proposed concept, each EV has one processing unit with the capability to process information in real-time. This information can be stored in a memory card and transmitted over the cloud via internet with an appropriate communication technology. The type of data transmitted by each vehicle could be the EVs ' location, time of charge, energy charged, charged rate and trip consumptions (see Fig. 1).

Figure 1.

To the best of our knowledge this problem has not yet been addressed in the context of a smart city in the literature. The developed work to obtain EVs' scenarios are either limited or problem-specific, usually as a complement to a certain developed application, e.g. an energy management optimization model. Indeed, it was not possible to find a work that focus solely on this issue. Nevertheless, the studied works [1,24,26,27,29] do not take into account the existence of a historic database, while aggregated representative fleets are considered, thus not obtaining individual EV's profiles. Estimating individual profiles can be important for energy resources scheduling in smart grids [32]. The work in [25], considers individual profiles, but does not propose to estimate locations as the purpose is to analyze the impact on the household consumption, whose location is a variable previously known. In fact, the uncertain location of EVs using MCS was not take into account in many papers [1,24-26,28].

The work presented in this paper, discusses a possible database taking into account the smart city vision and proposes methods that benefit from the use of historical data. In addition, the methods developed in this work enable to deal and obtain either an individual 
or a fleet profile, namely estimations regarding energy demand, location and time of connection.

\subsection{Organization of the paper}

After this introductory section, section II presents the proposed methods, section III presents an illustrative case study that considers a $30 \mathrm{EVs}$ fleet. Finally, the most relevant conclusions are presented in section IV.

\section{Proposed Methods}

The proposed stochastic scenario generation framework for day-ahead and hour-ahead horizon is discussed in this section. Moreover, the methods and their implementation to achieve the proposed objectives are also described, namely the MCS and the fuzzy logic approach.

\subsection{Stochastic Scenario Generation}

Fig. 2 shows an overview of the proposed EV's stochastic scenario generation. A dayahead and an hour-ahead stochastic scenario generation is proposed here. The day-ahead generation provides information about the EV's location during the next day (scenarios), considering the historical data contained in the cloud. The hour-ahead scenario takes into account the actual EV's location, and uses the historical data with similar pattern to generate a set of possible scenarios for the next hour. Day-ahead generation is designed to run once a day, while hour-ahead is meant to run at each hour. This information can be used by grid operators in the context of their operation tasks.

Figure 2. 
To achieve the mentioned tasks, it is necessary to identify which cloud data (big data) is relevant to be retrieved and what are the most adequate methods. Fig. 3 depicts the proposed approach, using MCS and FL.

Figure 3.

The Monte Carlo method is based on random sampling. In this sampling technique, a high number of random numbers are generated to estimate the probabilities of different system states [33]. This method is used in a wide range of applications, due to its stochastic nature, to obtain approximations for complex functions that are difficult to be analytically modelled. Assuming a database existence with a reasonable amount of historical data to run the MCS method, the necessary values must be filtered (e.g. vehicles' locations). The algorithm reduces the historical data to 4 periods representing 6 hours each. Reducing the data amount is an important aspect for the efficient use of this method. Otherwise, a very significant processing capacity is required. A matrix is built with the stored values regarding historical connections to the grid (for instance outlet/network bus) of a given EV. In this case, the historical data can belong to two different groups: working days and weekends. If the objective is to obtain system states for weekdays, then the selected data should be properly aligned with this objective and the historical data of weekdays should be filtered.

To obtain the energy demand uncertainty, a FL method is used, as well as a distribution function that best represents the EVs' demand. The probability distribution function that best represents the EVs' demand is obtained by combining a normal distribution [34] with the fuzzy membership function. To apply the FL, it is necessary to obtain historical data that incorporates the EVs' charging information, to define upper and lower bound values, and the number of degrees of truth. The fuzzy function centroid indicates for the shortest distance location for all points of the triangle, i.e. the average point of the area. 


\subsection{Monte Carlo Simulation}

The Monte Carlo is used in random and repetitive events, and its' main developer was the Hungarian-American mathematician John Von Neumann [35]. During World War II, in the atomic bomb construction project, Ulam, Von Neumann and Fermi considered this theory, which consisted of the direct simulation of probabilistic problems related to the neutron particles diffusion, when subjected to a nuclear fission process.

The MCS ideology portrays a statistical method used in stochastic problems. It is used in a wide range of applications in several areas such as: physics, mathematics and biology. The Monte Carlo model is often used in situations requiring to obtain numerical approximations to complex functions, for which it is not feasible or even impossible to achieve an analytical or deterministic solution. Monte Carlo methods are used mainly in three distinct classes: optimization problems, numerical integration and generating samples from a probability distribution.

Fig. 4 depicts the flowchart of MCS implemented in this paper. Assuming the existence of a database with a reasonable amount of historical data (as seen in Fig. 4), the necessary values needed to run the MCS methodology are prepared and assembled. A matrix is built with the stored values regarding historical connections to the grid (for instance outlet/network bus) of a given EV. The algorithm reduces the historical data from 24 periods ( 1 hour per period) to 4 periods, representing 6 hours each. Without this reduction process, the problem requires higher computational means, while the reduced data is still sufficient to represent the daily vehicles behavior [36]. The selected data is an important aspect of the correct use of the methodology, e.g. if the objective is to obtain system states for weekdays, then the selected 
data should be properly aligned with this objective and correspondingly filtering historical data that contains weekdays only.

The matrix can contain several entries corresponding each row to a different EV, each column to a different period and each third-dimension to a different day. With the appropriate records stored in the matrix, it is possible to evaluate another matrix, prob, storing the probabilities of being connected to a given network bus in a given period of the day. Hence, the MCS method uses this prob matrix to compare with random numbers and obtain each EV states. The logical comparison results in 0 or 1 corresponding to the state of connection to the grid, in matrix $M$. The location of connection in the grid is also associated in this stage, in matrix $M \_n$.

Accuracy in Monte Carlo Simulation

Figure 4.

According to [37] the accuracy level $(\beta)$ can be obtained by using equation (1):

$$
\beta=\sqrt{\frac{1-P}{N \cdot P}}
$$

The value of $P$ is the reference probability and the value of $N$ is the number of samples in MCS. In this specific case the reference probability $(P)$ corresponds to the probability of a given $\mathrm{EV}$ to follow the expected timetable and the expected journey without having breakdowns. Equation (2) shows how to obtain the reference probability, with the value of $x_{i}$ $=0$, representing a state where an EV is not charging and the value of $x_{i}=1$ representing a state where an EV is charging.

$$
P=\frac{1}{N} \sum_{i=1}^{N} x_{i}
$$

where: 
$P$ is the reference probability

$N$ is the number of MCS samples (number of runs)

$x_{i}$ is the state variable $(0 / 1)$

Equation (3) is obtained using (1):

$$
N=\frac{1-P}{\beta^{2} \cdot P}
$$

For a desired level of accuracy $(\beta)$ the number of MCS samples $(N)$ depends on the reference probability but they are independent on the problem dimensionality. As the number of MCS iterations increases, the accuracy level should improve towards a constant value, which tends be close to 0 .

\subsection{Fuzzy Logic}

The meaning of "fuzzy" term can be defined by something vague or uncertain [38]. FL is a very convenient approach for representing some forms of uncertainty, because it is based on a logic that recognizes more than simple true and false values. FL is often used to facilitate the expression of rules and facts. With FL, this propositions can be represented with degrees of truth. For example, the statement, "today is sunny", might be $100 \%$ true if there are no clouds, $80 \%$ true if there are a few clouds, $50 \%$ true if it's hazy and $0 \%$ true if it is rains all day. FL allows an infinite range of values in the range $[0,1]$, which would indicate the possibility of a statement to be true (1) or false (0), assuming intermediate logical values neither completely true nor false. The process of converting a fuzzy region in a final numeric value is designated by defuzzification and consist in simply evaluating the region center of gravity as expressed by (4): 


$$
v=\frac{\int_{x}(x \times t(x))}{\int_{x}(t(x))}
$$

where:

$v$ - defuzzification value

$t(x)$ - degree of truth in the point $\mathrm{x}$

From the historical database (big data), the energy consumption parameter was obtained, corresponding to the energy dispended in the vehicle trips. When the vehicle is charging, the consumption has a null value. To apply the FL approach it is necessary to evaluate the average energy consumption from the filtered data. The FL implementation, requires defining an upper bound, and lower bound values as well as the number of degrees of truth. The fuzzy function centroid corresponds to the shortest distance for the all points the resulting function figure. Fuzzification was applied to the average and standard deviation values.

The distribution that best represents the consumption of EVs is the normal distribution [34]. The normal distribution can be obtained using (5):

where:

$$
f p(P)=\frac{1}{\sqrt{2 \pi \times \sigma^{2}}} \times e^{\frac{-(P-\mu)^{2}}{2 \times \sigma^{2}}}
$$

$\mu$-represents the average value of consumption;

$\sigma$ - represents the standard deviation of consumption;

$P$-represents values resulting from the fuzzy function; 
With the fuzzified values previously evaluated the normal distribution functions were derived. In this distribution the average value and the standard deviation are the respective FL values. As a final result the interval of probability (pessimistic and optimistic) of a given EV is obtained, corresponding to a specific demand in a time period. This can be used by operators to improve grid planning and to understand the behavior of EVs customers.

\section{Case Study}

Currently there is no historical data in significant amounts related with EVs, e.g. location, charged energy and time of charging. Hence, a scenario was generated concerning a realistic study, using a computer application in MATLAB software for a $30 \mathrm{EV}$ sleet. Fig. 5 shows the radial distribution network configuration and the Charging Stations (CS) location, where the EVs fleet can charge during the day. The case study considered a medium voltage distribution network (a voltage of $12.66 \mathrm{kV}$ ) with 33 buses. The Charging Stations (CS) are located at buses 10, 16, and 26, as represented in Fig. 5.

Figure 5.

Due to the differences in the behavior of the EV users during weekdays and weekends, the historical data is divided in two sets. The weekdays' group set has a total of 254 days, while the weekends' and holidays' group set correspond to a total of 111 days. Aspects such as: the probability of the EVs' early leaving, the probability for breakdown and the probability of choosing the charging location are also considered in the model. The program considers the possible changes in the planned route, i.e. breakdowns or failures in the EV fleet, therefore it simulates a realistic behavior. The program stores the data in a permanent database, constituting the starting point for the MCS. This data could be considered the big data (see Fig. 1). 


\subsection{Day-ahead}

The information filtered from the data corresponds to the EV's locations, i.e., for each EV and for each day period, it is possible to identify the bus where the EV was connected to the grid. To enable a feasible computation processing, one algorithm to reduce the amount of data was implemented. This algorithm compiles the historical data from 24 to 4 periods, where each period represents 6 hours. The first period corresponds to $1 \mathrm{~h}-6 \mathrm{~h}$, the second period to $7 \mathrm{~h}-12 \mathrm{~h}$, the third period to $13 \mathrm{~h}-18 \mathrm{~h}$ and the last period to $19 \mathrm{~h}-24 \mathrm{~h}$. The chosen periods are sufficient to represent the daily behavior of a vehicle. The algorithm to reduce the scenarios employed statistics techniques, such as the mode. With the reduced data, the probability of each EV to be connected to a given bus at a certain period was evaluated. The result was a probabilities matrix that indicated for each EV the probability of being connected to a certain bus, in a given period. This data treatment enabled to use acceptable computational resources. The probability matrix was used as an input to the MCS method to compare with random numbers and to obtain each EV's states. The outputs were a logical value, 1 and 0 . If the value was 1 , it meant that the EV was connected. After running MCS, the repeated states were counted and sorted. This resulted in a matrix containing the states and number of times that a given state occurred. More details about the method can be seen in the section 2.2.

Considering the specific case of the weekday's scenario, some results are described here. The MCS was simulated for 2 million iterations corresponding in practice to 2 million possible days. The result corresponds to $1,816,334$ of different possible states. However, a lot of those states had a very low probability, nearly zero. Hence, those states were discarded, leaving only 10 states to consider. The probability of the top 3 and the other states ( 7 of 10) can be seen in Fig. 6. 
Figure 6.

Analyzing State 1 (Scenario 1), which is the most probable state to occur, the results showed that there are some EVs with the same location patterns. A total of 4 groups were created. The vehicles that belong to each group are depicted in table I. These groups were obtained by analyzing each individual EV's profile. If they matched, e.g. same profile, they were aggregated. In this case, group 1 is the group with the higher number of EVs. A clustering method or other grouping technique can be used to find similarities. Table II presents the network locations (see figure 5) for these 4 groups. Figure 7 illustrates the location for the groups identified in state 1 . This figure presents the profile of state 1 obtained with MCS.

Table 1.

Table 2.

Figure 7.

To determine the degree of precision, which aims to quantify the number of MCS iterations needed to obtain results with acceptable accuracy, equation (1) was used. In this case $P$ is taken as the probability of state 1 , as this is the reference state, the one that should occur more often. To verify if 2 million iterations are enough for this study, a script program has been executed starting with 100,000 iterations and steadily increasing this number by 100,000 until $2,000,000$. In each increment the value of accuracy was determined using the reference probability. Fig. 8 presents the degree of precision obtained for each increment from 100,000 to $2,000,000$ with a step of 100,000 . As illustrated by this figure, as the number of iterations is increased, the MCS accuracy level improves.

Figure 8. 
The simulations were performed in a computer with an Intel Xeon W3550 $3.07 \mathrm{GHz}$ processor with $12 \mathrm{~GB}$ of RAM and Windows 8.1. The total execution time to run the MCS for this case study with 30 EVs was 262 seconds. The RAM used for execution of this process was approximately $8.5 \mathrm{~GB}$.

\subsection{Hour-ahead}

In this section the results for the hour-ahead are presented. The hour-ahead takes into account the actual EV location and it uses historical data with similar pattern to generate a set of possible scenarios for the next hour. A prediction for the hour 1 of day 254 was made based on the location and previous historical data. The algorithm developed in this work looked for similar location patterns in the historical data and returned the possible locations for the next hour based on the previous data. With this information, each EV probability to be connected to a given bus was evaluated. The MCS was simulated for 2 million iterations, to determine a relevant set of possible states, thus obtaining a prediction of the next location for the next hour, in this specific case, hour 1 of day 254. The result after evaluating MCS was 216,170 of possible different states. However, a lot of those states had a very low probability, nearly zero. Hence, theses states were discarded, leaving only 10 states to consider. The probability of the top 3 states corresponds to $76 \%$, wherein the first state represents $66 \%$ probability of occurrence, the second one $6 \%$ and the third $4 \%$. The other states ( 7 of 10 ) corresponds to a total probability of $12 \%$.

In Fig. 9 it is possible to see the EVs predictable behavior for the next hour (for the most likely state: 1), which in this case corresponds to the hour 1 of day 254 , taking into account the actual location for the hour 24 of the day 253 . The probability of this locations for the 
EVs in the next hour is $66 \%$.

Figure 9. The hour-ahead procedure was performed for the remaining hours, of day 254. Fig. 10 shows the EV-9 behavior for the 24 hours of day 254, for the top 3 states (colored dashed lines), which are the most probable states of occurrence. It is possible to see that in the top 3 states, the EV-9 as a high probability to be in trip between $7-13 \mathrm{~h}$ and $21-24 \mathrm{~h}$ periods. Hence, it is certain that the EV cannot charge in these periods. In addition, during the night there is a considerable amount of probability that EV-9 can be on bus 10 between $2 \mathrm{~h}$ and $3 \mathrm{~h}$ (state 2 ), with less probability to be on the same bus 10 between $4-5 \mathrm{~h}$, according to state 3 .

Figure 10.

To evaluate the degree of precision, which aims to quantify the number of iterations needed in MCS to obtain results with an acceptable error, equation (1) was used and the process realized in day-ahead was repeated.

\subsection{Energy Demand}

Regarding the uncertainty analysis associated with the energy demand, and considering the specific case of EV-1 some results are presented. Initially, the average of energy demand per trip was evaluated for a period of 24 hours a day (the average value for each hour along of 254 days), which can be seen in Fig. 11 where it is possible to observe the consumption variations for each hour. After determining the average, the value of consumption standard deviation was evaluated, as shown in Fig. 12.

Figure 11.

Figure 12.

FL was applied both for the average and standard deviation values. To implement the FL triangular function the lower bound, the upper bound, and the degrees of truth were defined. 
To a realistic result, two different cases were considered, one optimistic case and one pessimist case. For the pessimistic case, a lower bound with a value of $-10 \%$ and an upper bound with a value of $20 \%$ was used. For the optimistic case, lower bound with a value of $20 \%$ and an upper bound with a value of $10 \%$ was used.

The results of the fuzzy method obtained for EV-1 in the hour 2 can be seen in the table III and IV. Table III represents the fuzzy for the energy demand average value and table IV depicts the energy demand standard deviation. Both tables represent the values of EV-1 in the hour 2 .

Table 3.

Table 4.

The representation of the fuzzy logic for the average can be seen it in the Fig. 13 (pessimistic case) and Fig. 14 (optimistic case).

Figure 13.

Figure 14.

In the view of these results, a probable energy demand range has been obtained, that can vary between 47.01 and $72.91 \mathrm{~kW}$, depending on the considered deviations.

The next stage was to implement the normal distribution with the values that were obtained with the FL (average fuzzy values and standard deviation fuzzy values).

Fig. 15 depicts the probability distribution functions for each case, namely the original normal distribution, the fuzzy optimistic and the fuzzy pessimistic. The fuzzy optimistic and fuzzy pessimistic, resulted in the calculation of the normal distribution with the fuzzy values. The normal distribution, considered the original values for average and standard deviation. The values presented regard the demand probabilistic distribution for EV-1 in hour 2. 
Figure 15.

With the proposed fuzzy-probabilistic method it is possible to obtain the probabilities' range that a given energy demand might reach. For example, the probability of EV-1 to have a charging demand higher than $20 \mathrm{kWh}$ over 24 hour can be verified in Fig. 16 for the optimistic case and pessimistic case, respectively. 


\section{Conclusions}

This paper presents a framework to estimate the EVs' possible states regarding their location and periods of connection in the grid. The MCS is used to model the uncertainties related with the location of EVs in the grid. The proposed model assumes the existence of efficient communication/information systems and cloud computing capabilities in the big data context. The energy demand is also estimated with the fuzzy approach. A numerical example is shown using a fleet of Electric Vehicles (EV) considering a smart city environment to obtain the states of EVs, regarding their location in the grid. In this paper one algorithm to reduce the number of scenarios and aggregate the data was implemented to enable a feasible computation by optimizing the algorithm execution time. Without implementing the data reduction approach, the computational resources required for executing the code would be very high and not efficient for the day-ahead time window. In future, it is recommend to try other alternatives of scenario generation techniques, e.g. Bootstrap method to reduce computational burden and make the idea more scalable.

\section{Acknowledgements}

This work is supported by FEDER Funds through COMPETE program and by

National Funds through FCT under the projects FCOMP-01-0124-FEDER: PEstOE/EEI/UI0760/2014, and PTDC/SEN-ENR/122174/2010, and SFRH/BD/87809/2012.

\section{References}

[1] Božič D, Pantoš M. Impact of electric-drive vehicles on power system reliability. Energy 2015;83:511-20. doi:10.1016/j.energy.2015.02.055. 
[2] Chan CC. The State of the Art of Electric, Hybrid, and Fuel Cell Vehicles. Proc IEEE 2007;95:704-18. doi:10.1109/JPROC.2007.892489.

[3] Khaligh A. Battery, Ultracapacitor, Fuel Cell, and Hybrid Energy Storage Systems for Electric, Hybrid Electric, Fuel Cell, and Plug-In Hybrid Electric Vehicles: State of the Art. IEEE Trans Veh Technol 2010;59:2806-14. doi:10.1109/TVT.2010.2047877.

[4] Saxena S, MacDonald J, Moura S. Charging ahead on the transition to electric vehicles with standard 120V wall outlets. Appl Energy 2015;157:720-8. doi:10.1016/j.apenergy.2015.05.005.

[5] Emadi A, Rajashekara K. Power Electronics and Motor Drives in Electric, Hybrid Electric, and Plug-In Hybrid Electric Vehicles. IEEE Trans Ind Electron 2008;55:2237-45. doi:10.1109/TIE.2008.922768.

[6] Li F, Qiao W, Sun H, Wan H, Wang J, Xia Y, et al. Smart Transmission Grid: Vision and Framework. IEEE Trans Smart Grid 2010;1:168-77. doi:10.1109/TSG.2010.2053726.

[7] Galus MD, Waraich RÁ, Noembrini F, Steurs K, Georges G, Boulouchos K, et al. Integrating Power Systems, Transport Systems and Vehicle Technology for Electric Mobility Impact Assessment and Efficient Control. Smart Grid, IEEE Trans 2012;3:934-49. doi:10.1109/TSG.2012.2190628.

[8] Richardson DB. Electric vehicles and the electric grid: A review of modeling approaches, Impacts, and renewable energy integration. Renew Sustain Energy Rev 2013;19:247-54. 
[9] Cardoso G, Stadler M, Chehreghani Bozchalui M, Sharma R, Marnay C, BarbosaPovoa A, et al. Stochastic programming of vehicle to building interactions with uncertainty in PEVs driving for a medium office building. Ind. Electron. Soc. IECON 2013-39th Annu. Conf. IEEE, IEEE; 2013, p. 7648-53.

[10] Kempton W, Tomić J. Vehicle-to-grid power fundamentals: Calculating capacity and net revenue. J Power Sources 2005;144:268-79. doi:http://dx.doi.org/10.1016/j.jpowsour.2004.12.025.

[11] Galus MD, Zima M, Andersson G. On integration of plug-in hybrid electric vehicles into existing power system structures. Energy Policy 2010;38:6736-45.

[12] San Román TG, Momber I, Abbad MR, Sánchez Miralles Á. Regulatory framework and business models for charging plug-in electric vehicles: Infrastructure, agents, and commercial relationships. Energy Policy 2011;39:6360-75.

[13] Sortomme E, El-Sharkawi MA. Optimal Charging Strategies for Unidirectional Vehicle-to-Grid. IEEE Trans Smart Grid 2011;2:131-8. doi:10.1109/tsg.2010.2090910.

[14] Battistelli C, Baringo L, Conejo AJ. Optimal energy management of small electric energy systems including V2G facilities and renewable energy sources. Electr Power Syst Res 2012;92:50-9.

[15] Han S, Han S, Sezaki K. Development of an optimal vehicle-to-grid aggregator for frequency regulation. Smart Grid, IEEE Trans 2010;1:65-72.

[16] Sousa T, Morais H, Soares J, Vale Z. Day-ahead resource scheduling in smart grids 
considering Vehicle-to-Grid and network constraints. Appl Energy 2012;96:183-93. doi:10.1016/j.apenergy.2012.01.053.

[17] Batty M, Axhausen KW, Giannotti F, Pozdnoukhov A, Bazzani A, Wachowicz M, et al. Smart cities of the future. Eur Phys Journal-Special Top 2012;214:481.

[18] Balac N. "Green Machine” Intelligence: Greening and Sustaining Smart Grids. Intell Syst IEEE 2013;28:50-5. doi:10.1109/MIS.2013.127.

[19] Zhang S, Huang P, Sun Y. A multi-criterion renewable energy system design optimization for net zero energy buildings under uncertainties. Energy 2016;94:65465. doi:10.1016/j.energy.2015.11.044.

[20] Scott MJ, Daly DS, Hathaway JE, Lansing CS, Liu Y, McJeon HC, et al. Calculating impacts of energy standards on energy demand in U.S. buildings with uncertainty in an integrated assessment model. Energy 2015;90:1682-94. doi:10.1016/j.energy.2015.06.127.

[21] Kim S, Koo J, Lee CJ, Yoon ES. Optimization of Korean energy planning for sustainability considering uncertainties in learning rates and external factors. Energy 2012;44:126-34. doi:10.1016/j.energy.2012.02.062.

[22] Wang S, Zhang X, Liu L. Multiple stochastic correlations modeling for microgrid reliability and economic evaluation using pair-copula function. Int J Electr Power Energy Syst 2016;76:44-52. doi:10.1016/j.ijepes.2015.09.024.

[23] Nijhuis M, Gibescu M, Cobben JFG. Bottom-up Markov Chain Monte Carlo approach for scenario based residential load modelling with publicly available data. Energy 
Build 2016;112:121-9. doi:10.1016/j.enbuild.2015.12.004.

[24] Khazali A, Kalantar M. A stochastic-probabilistic energy and reserve market clearing scheme for smart power systems with plug-in electrical vehicles. Energy Convers Manag 2015;105:1046-58. doi:10.1016/j.enconman.2015.08.050.

[25] Neaimeh M, Wardle R, Jenkins AM, Yi J, Hill G, Lyons PF, et al. A probabilistic approach to combining smart meter and electric vehicle charging data to investigate distribution network impacts. Appl Energy 2015;157:688-98. doi:10.1016/j.apenergy.2015.01.144.

[26] Pantoš M. Stochastic optimal charging of electric-drive vehicles with renewable energy. Energy 2011;36:6567-76.

[27] Soares FJ, Peças Lopes JA, Almeida PMR. A Monte Carlo method to evaluate electric vehicles impacts in distribution networks. Innov. Technol. an Effic. Reliab. Electr. Supply (CITRES), 2010 IEEE Conf., 2010, p. 365-72. doi:10.1109/CITRES.2010.5619777.

[28] Shaaban MF, Atwa YM, El-Saadany EF. PEVs Modeling and Impacts Mitigation in Distribution Networks. Ieee Trans Power Syst 2013;28:1122-31. doi:Doi 10.1109/Tpwrs.2012.2212467.

[29] Garcia-Valle R, Vlachogiannis JG. Letter to the Editor: Electric Vehicle Demand Model for Load Flow Studies. Electr Power Components Syst 2009;37:577-82. doi:10.1080/15325000802599411.

[30] Vlachogiannis JG. Probabilistic Constrained Load Flow Considering Integration of 
Wind Power Generation and Electric Vehicles. IEEE Trans Power Syst 2009;24:1808-17. doi:10.1109/TPWRS.2009.2030420.

[31] Silva MA, de Melo HN, Trovao JP, Pereirinha PG, Jorge HM. An integrated fuzzy logic energy management for a dual-source electric vehicle. Ind. Electron. Soc. IECON 2013-39th Annu. Conf. IEEE, IEEE; 2013, p. 4564-9.

[32] Su W, Wang J, Roh J. Stochastic Energy Scheduling in Microgrids With Intermittent Renewable Energy Resources. IEEE Trans Smart Grid 2014;5:1876-83. doi:10.1109/TSG.2013.2280645.

[33] Rubinstein RY, Kroese DP. Simulation and the Monte Carlo method. vol. 707. John Wiley \& Sons; 2011.

[34] Zhao J, Wen F, Dong ZY, Xue Y, Wong KP. Optimal Dispatch of Electric Vehicles and Wind Power Using Enhanced Particle Swarm Optimization. IEEE Trans Ind Informatics 2012;8:889-99. doi:10.1109/TII.2012.2205398.

[35] Eckhardt R. Stan ulam, john von neumann, and the monte carlo method. Los Alamos Sci 1987;15:30.

[36] Neubauer J, Brooker A, Wood E. Sensitivity of plug-in hybrid electric vehicle economics to drive patterns, electric range, energy management, and charge strategies. J Power Sources 2013;236:357-64. doi:10.1016/j.jpowsour.2012.07.055.

[37] Canizes B, Soares J, Vale Z, Khodr HM. Hybrid fuzzy Monte Carlo technique for reliability assessment in transmission power systems. Energy 2012;45:1007-17. doi:DOI 10.1016/j.energy.2012.06.049. 
[38] Harris CJ, Moore CG, Brown M. Intelligent Control: Aspects of Fuzzy Logic and Neural Networks 1993.

[39] Baran ME, Wu FF. Network Reconfiguration in Distribution-Systems for Loss Reduction and Load Balancing. IEEE Trans Power Deliv 1989;4:1401-7. doi:Doi $10.1109 / 61.25627$. 
Figure Captions:

Fig.1. EVs in the IoT context: a conceptual view

Fig.2. EVs Stochastic Scenario Generation: day-ahead and hour-ahead

Fig.3. Proposed approach implemented

Fig.4. Flowchart of the implemented Monte Carlo Simulation

Fig.5. Medium voltage distribution network (adapted from [39])

Fig.6. Probability of states

Fig.7. Profile of state 1 (probability of $67 \%$ ) for the different groups

Fig. 8. Accuracy level of MCS

Fig.9. Behavior locations of the EVs for the hour 1

Fig.10. Top-3 states for the EV-9

Fig.11. EV-1 average energy demand

Fig.12. EV-1 standard deviation of energy demand

Fig.13. Representation of fuzzy for the average in EV-1 (Pessimistic Case)

Fig.14. Representation of fuzzy for the average in EV-1 (Optimistic Case)

Fig.15. Probability distribution functions

Fig.16. Probability of energy demand higher than $20 \mathrm{kWh}$ 
Tables Captions:

Table 1. Groups of EVs (State 1 - probability of 67\%)

Table 2. State 1 for the 4 groups (bus location)

Table 3. Average Fuzzy values for EV-1 in the hour 2 (kWh)

Table 4. Standard Deviation Fuzzy values for EV-1 in the hour $2(\mathrm{kWh})$ 


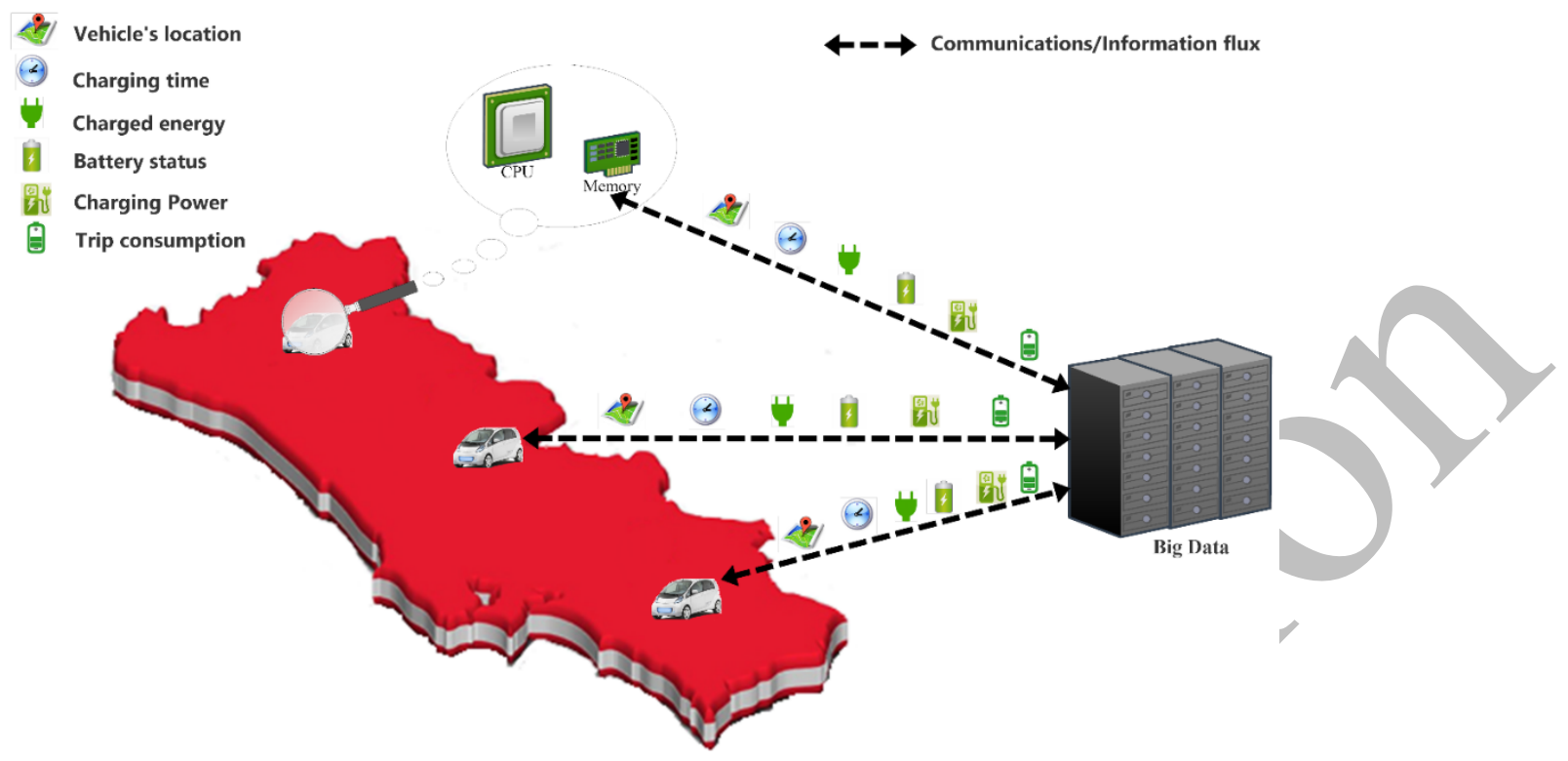

Fig.1. EVs in the IoT context: a conceptual view

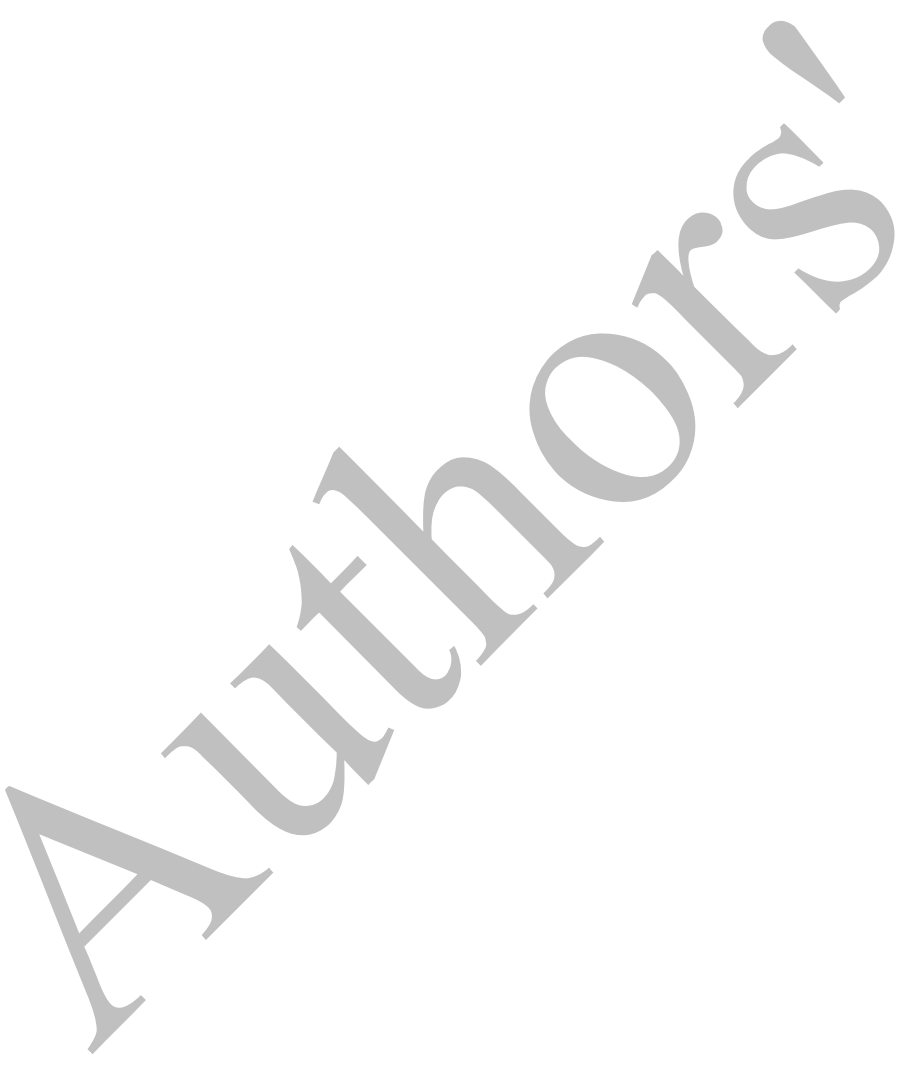




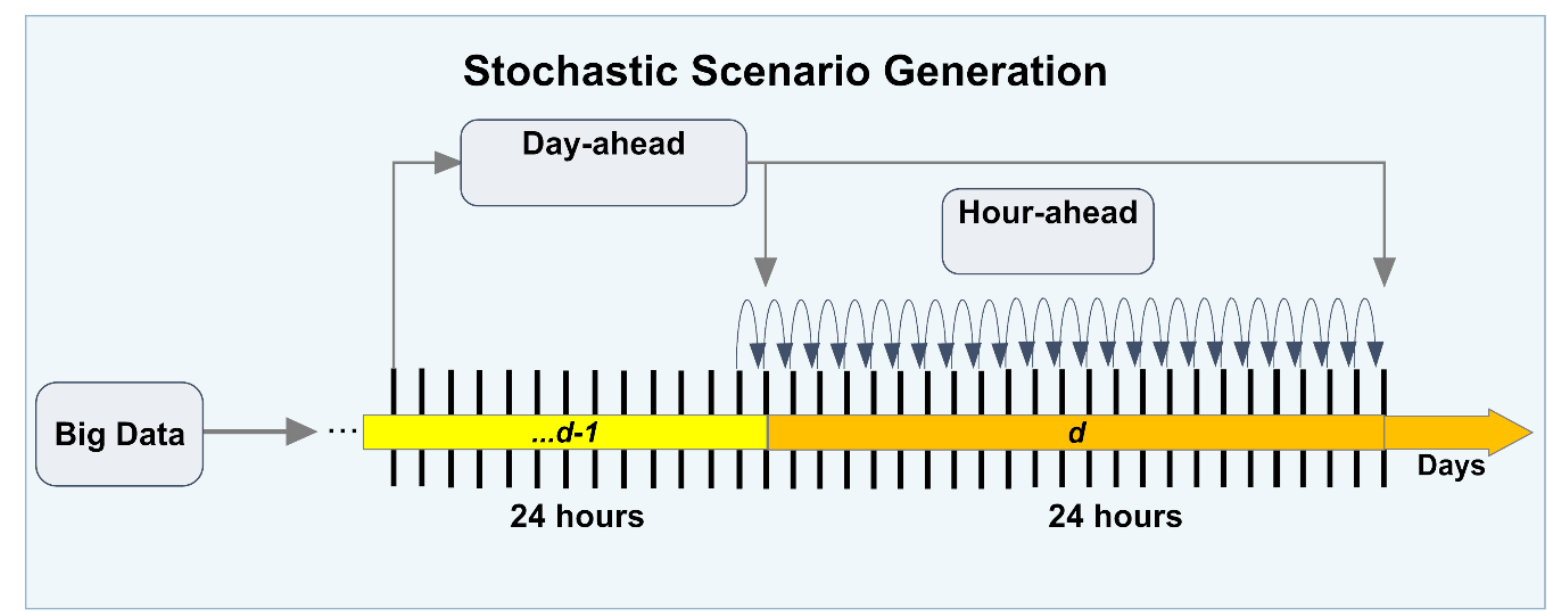

Fig.2. EVs Stochastic Generation: day-ahead and hour-ahead 


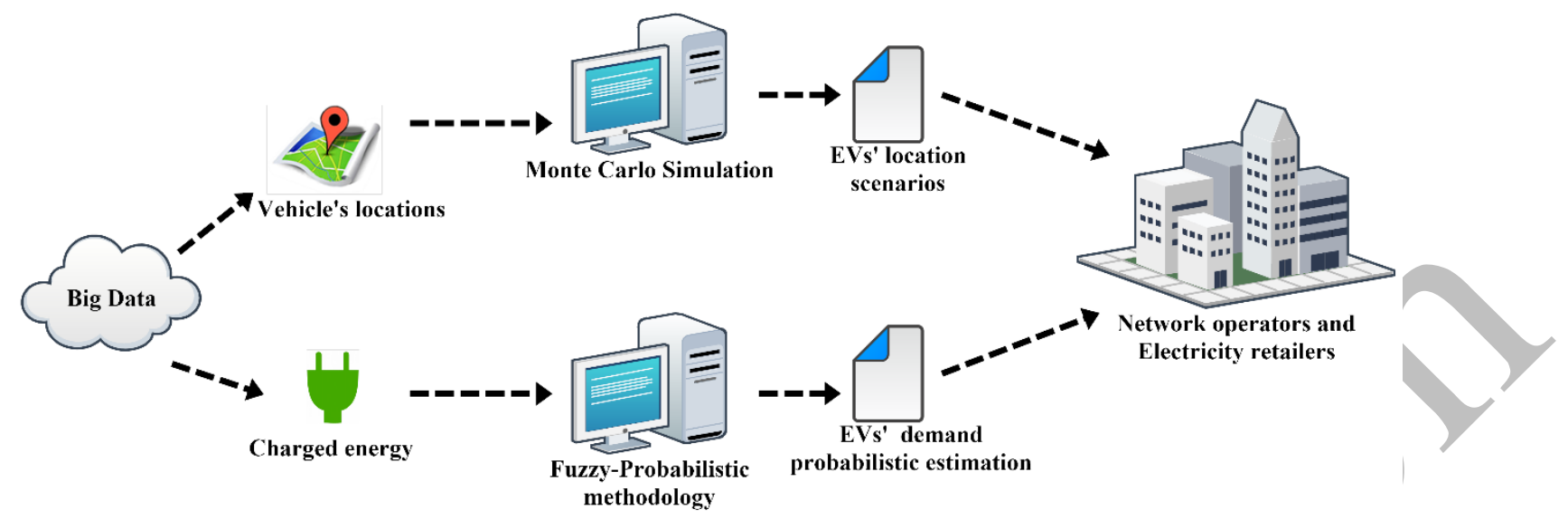

Fig.3. Proposed approach 


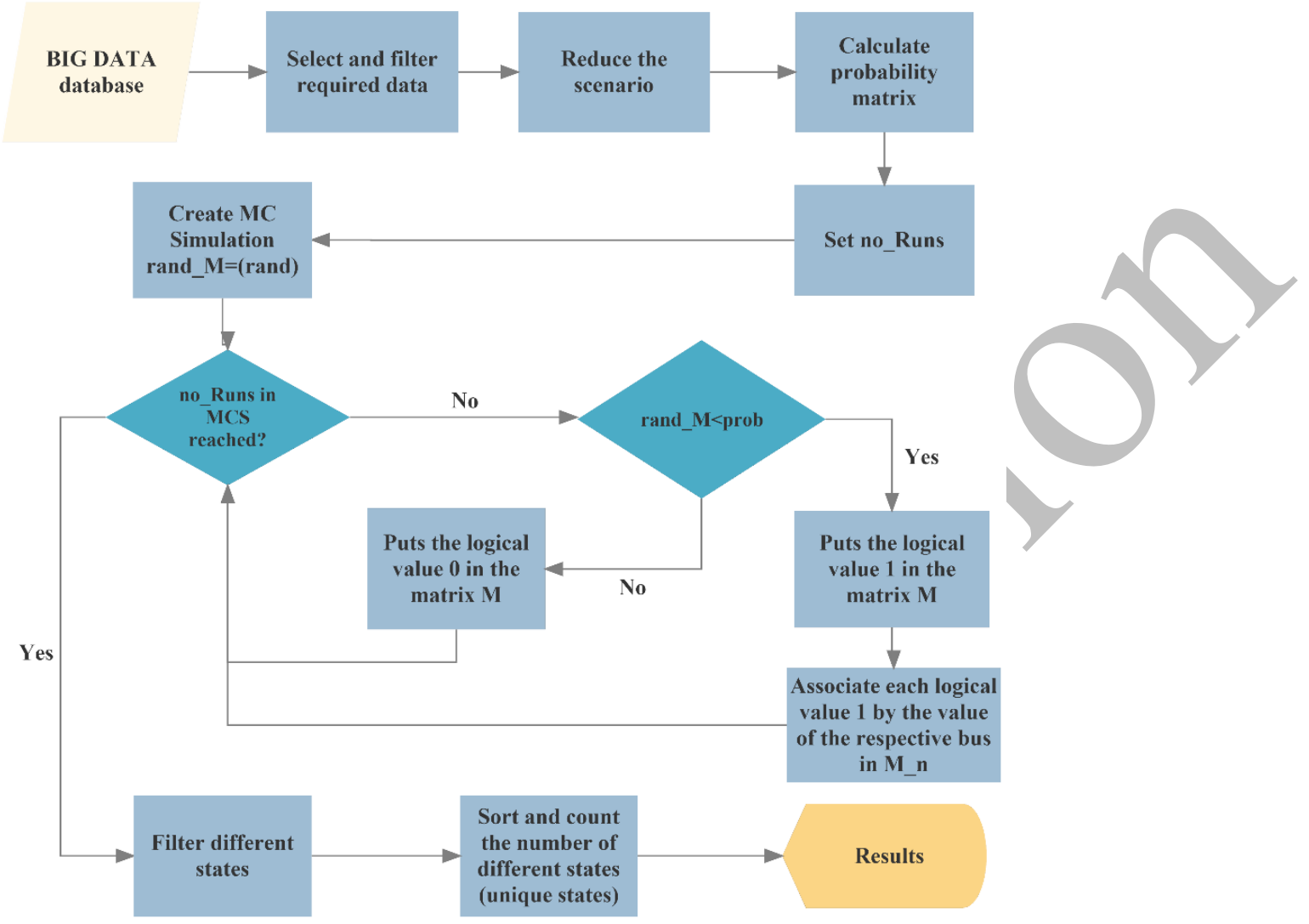

Fig.4. Flowchart of the implemented Monte Carlo Simulation 


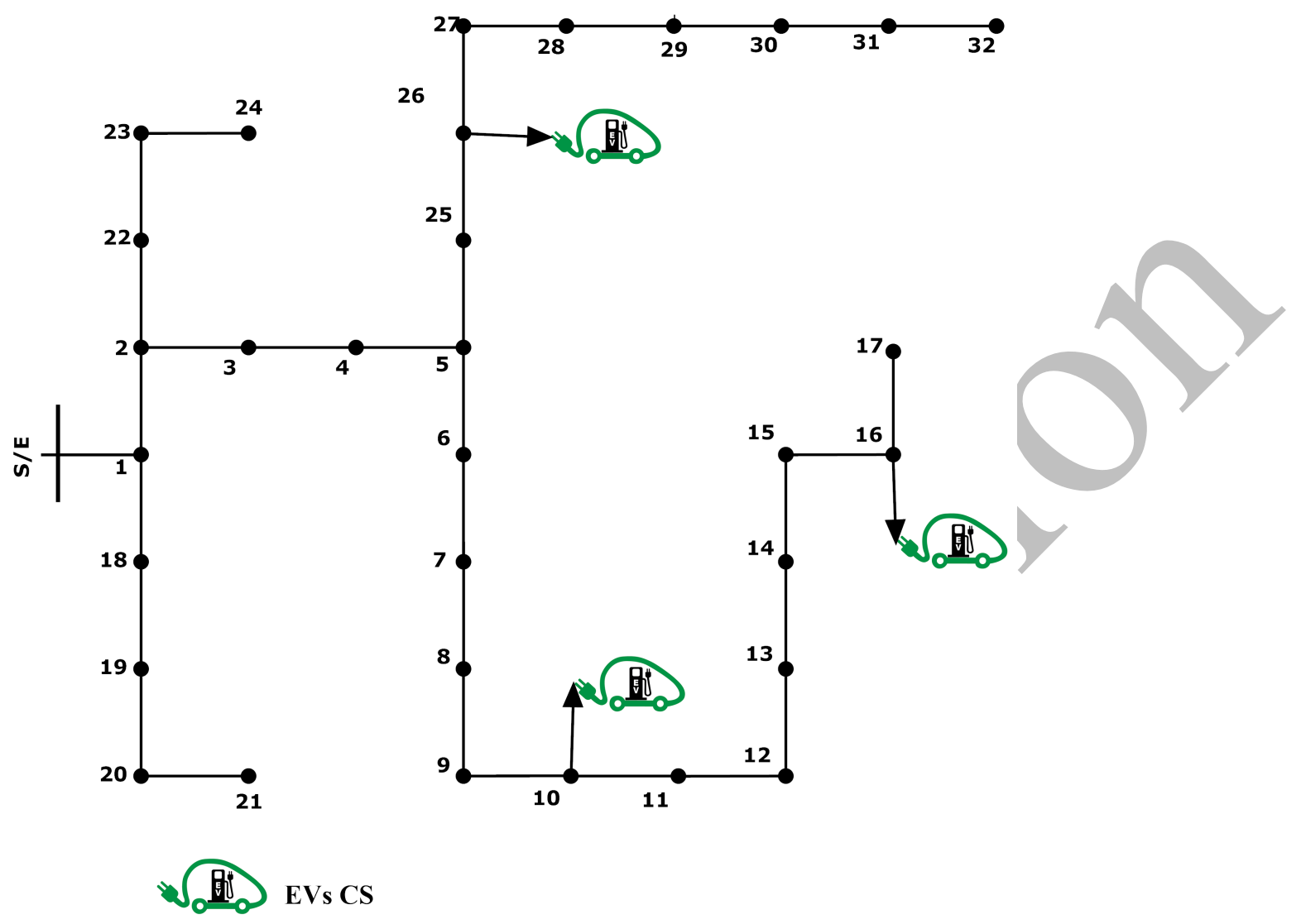

Fig.5. Medium voltage distribution network (adapted from [39]) 

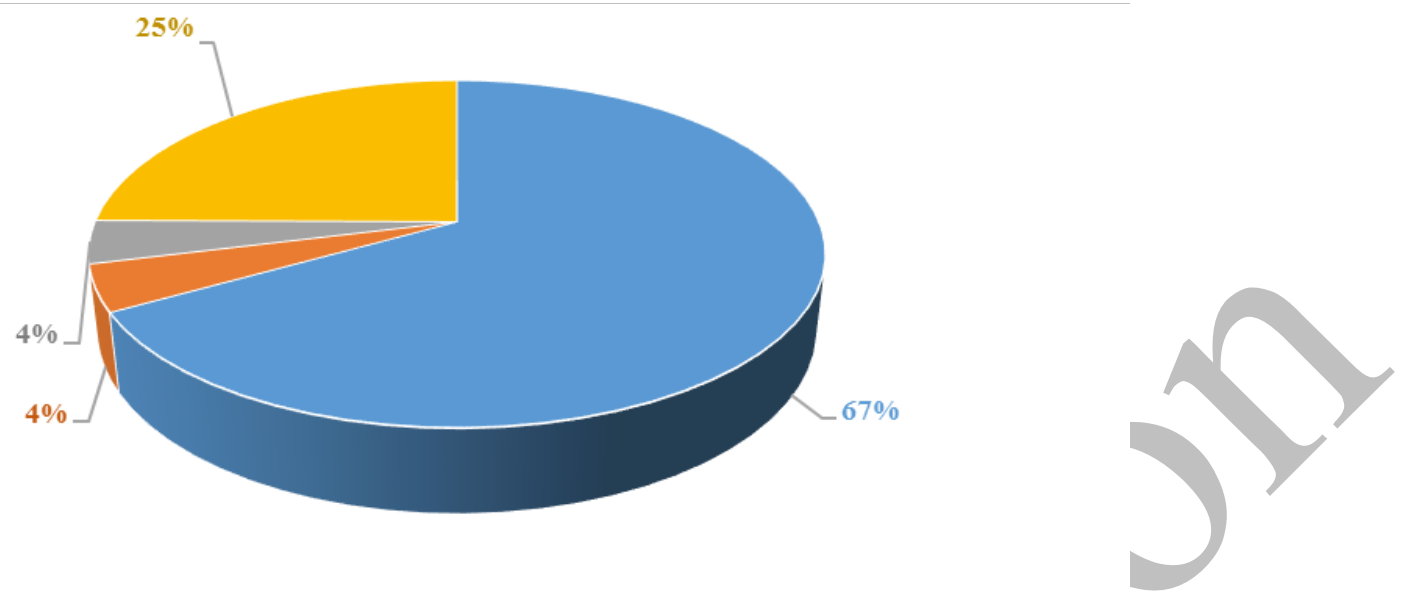

- State 1 -State 2 " State 3 = Other States

Fig.6. Probability of states

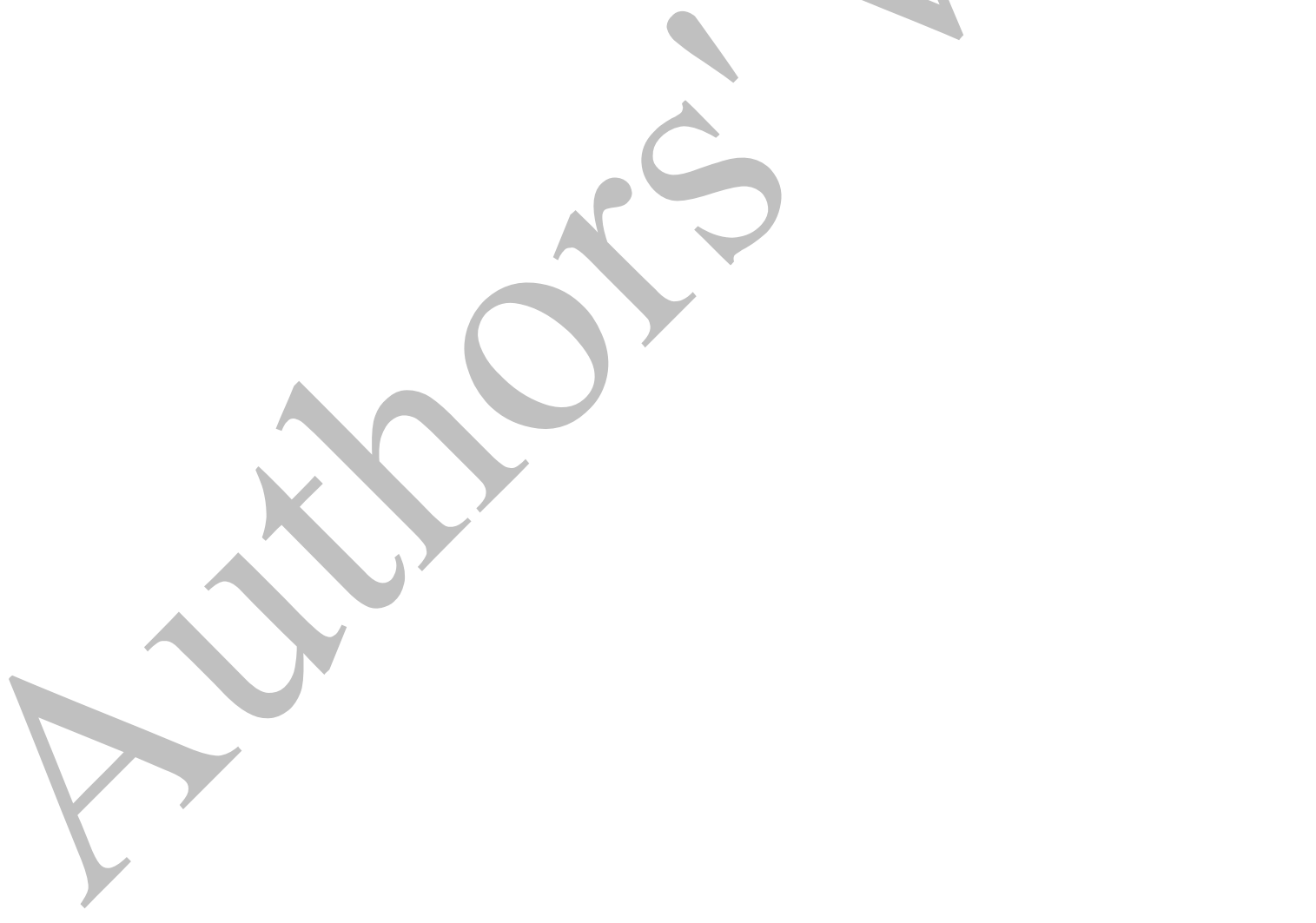




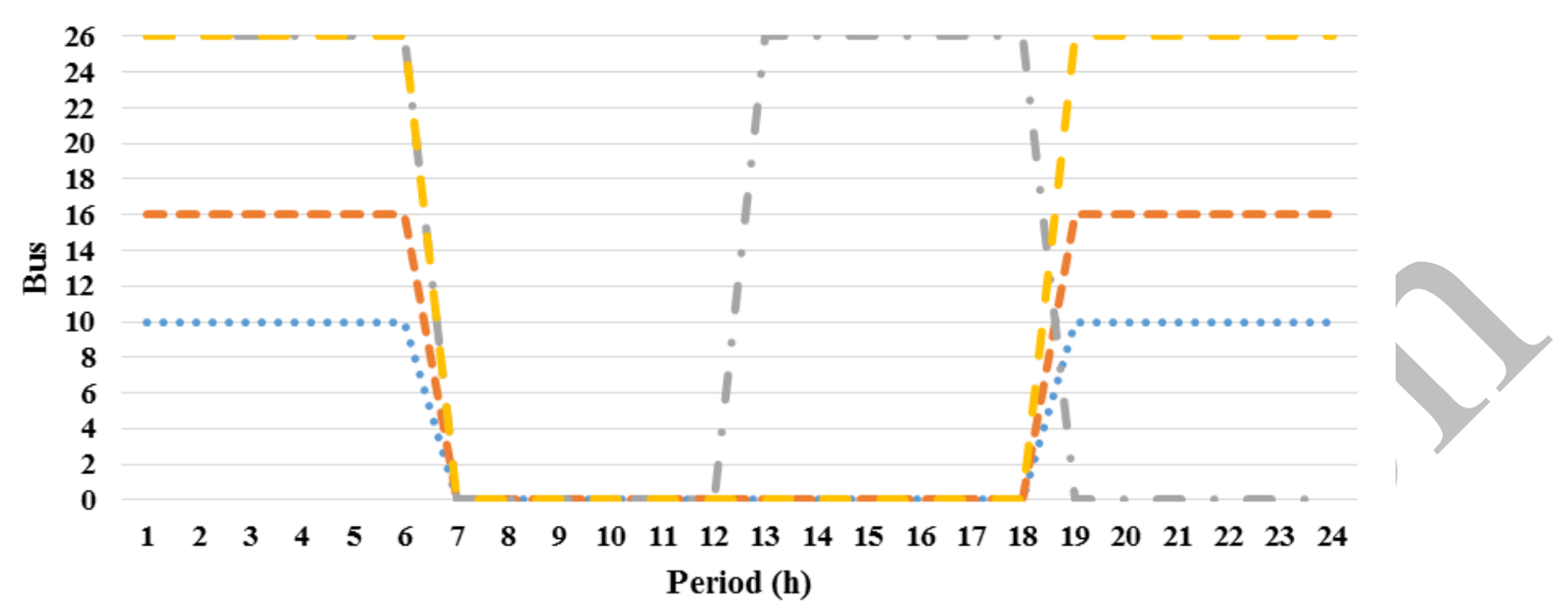

… Group $1--$ Group $2-$ Group $3-$ Group 4

Fig.7. Profile of state 1 (probability of $67 \%$ ) for the different groups 


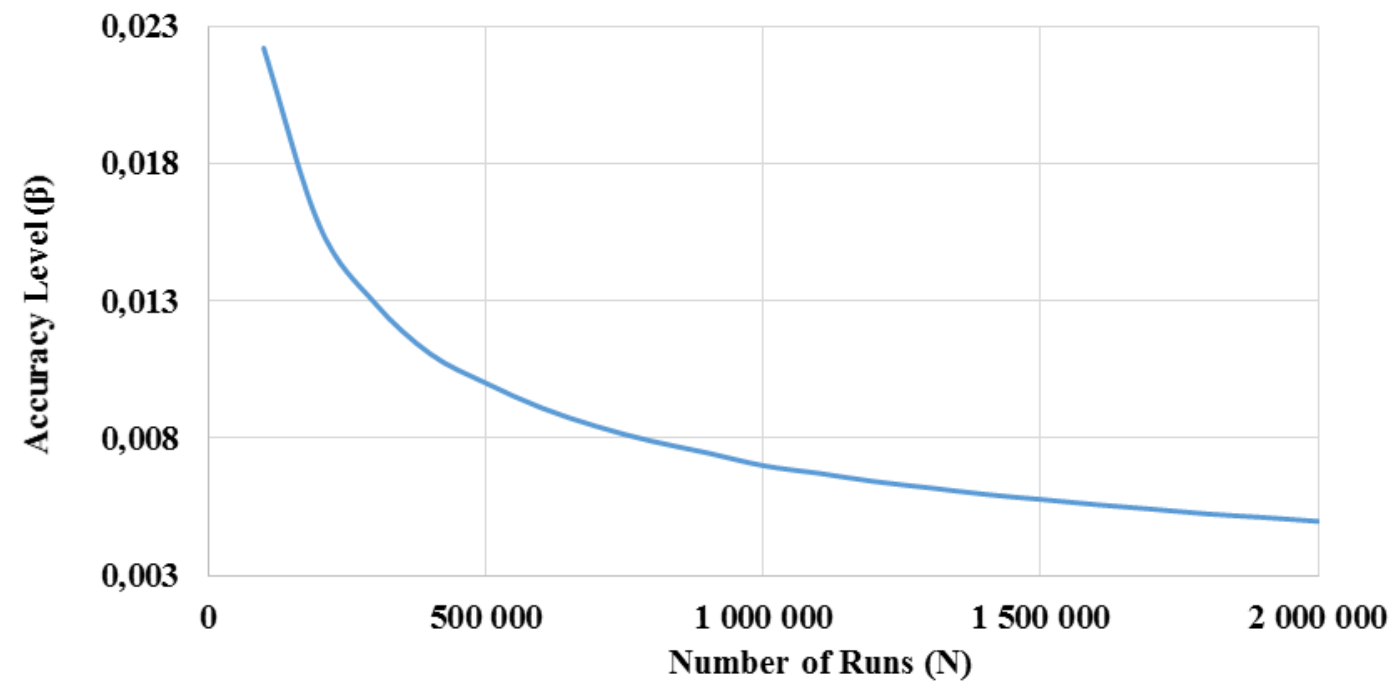

Fig.8. Accuracy level of MCS

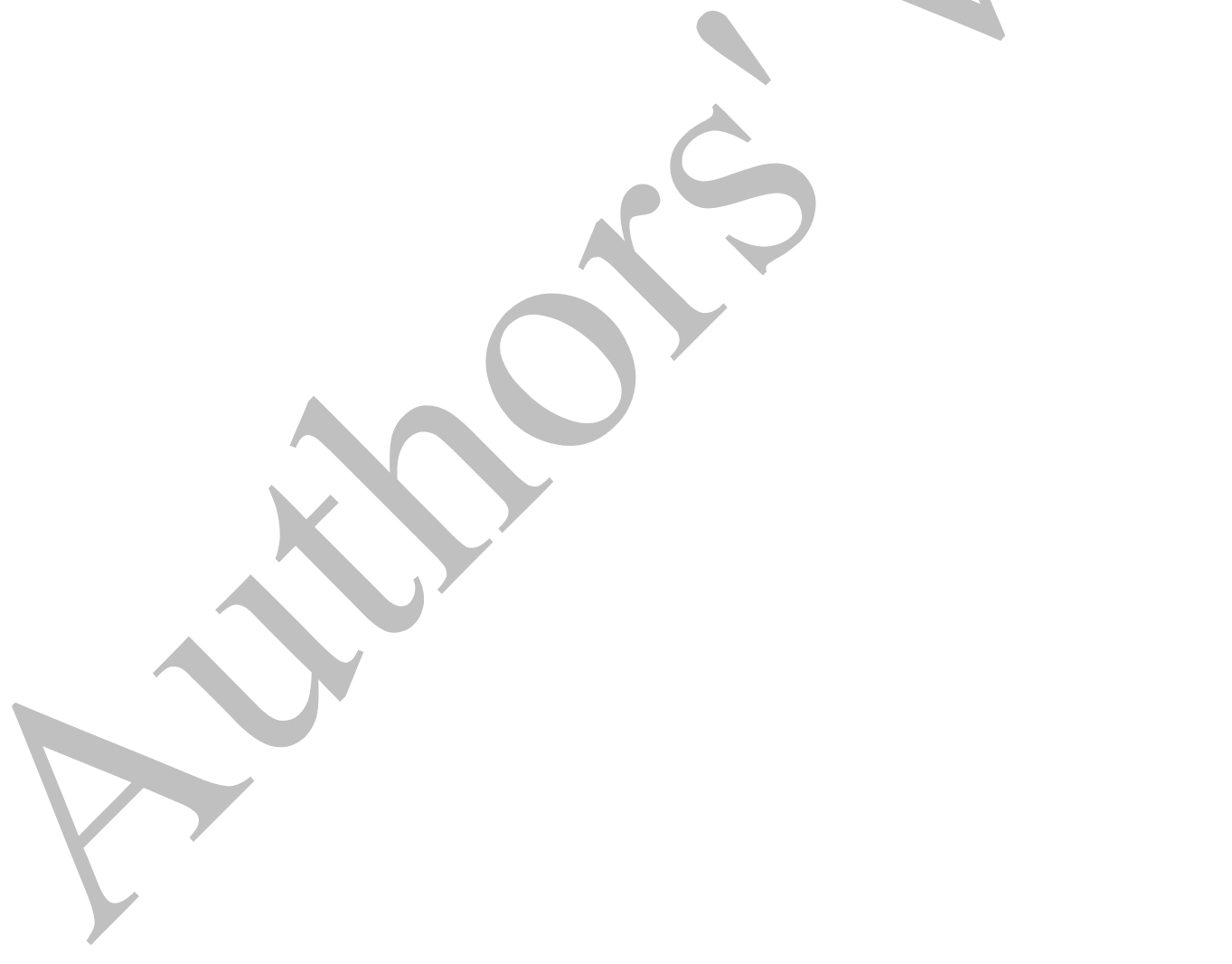




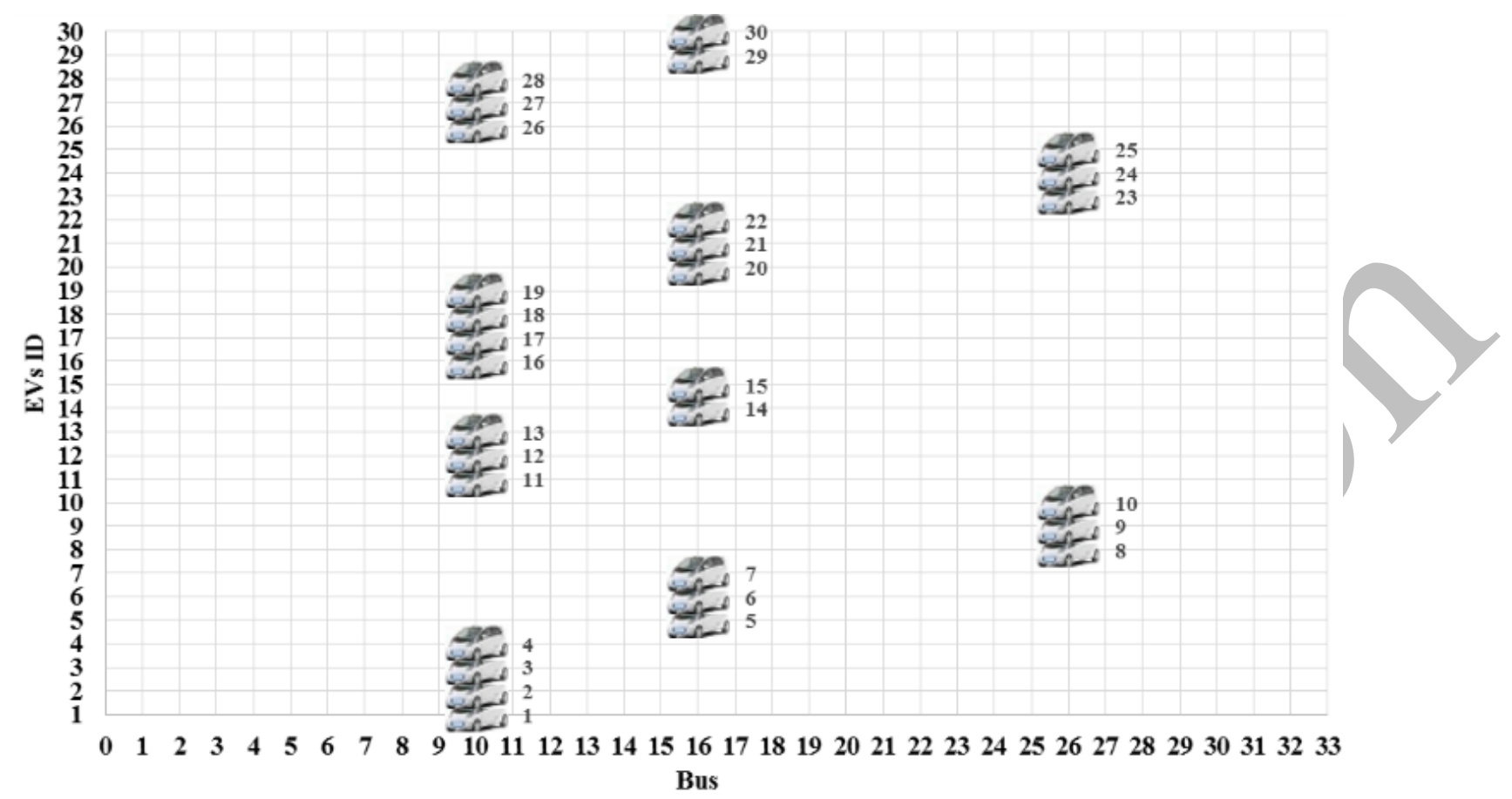

Fig.9. Behavior locations of the EVs for the hour 1 


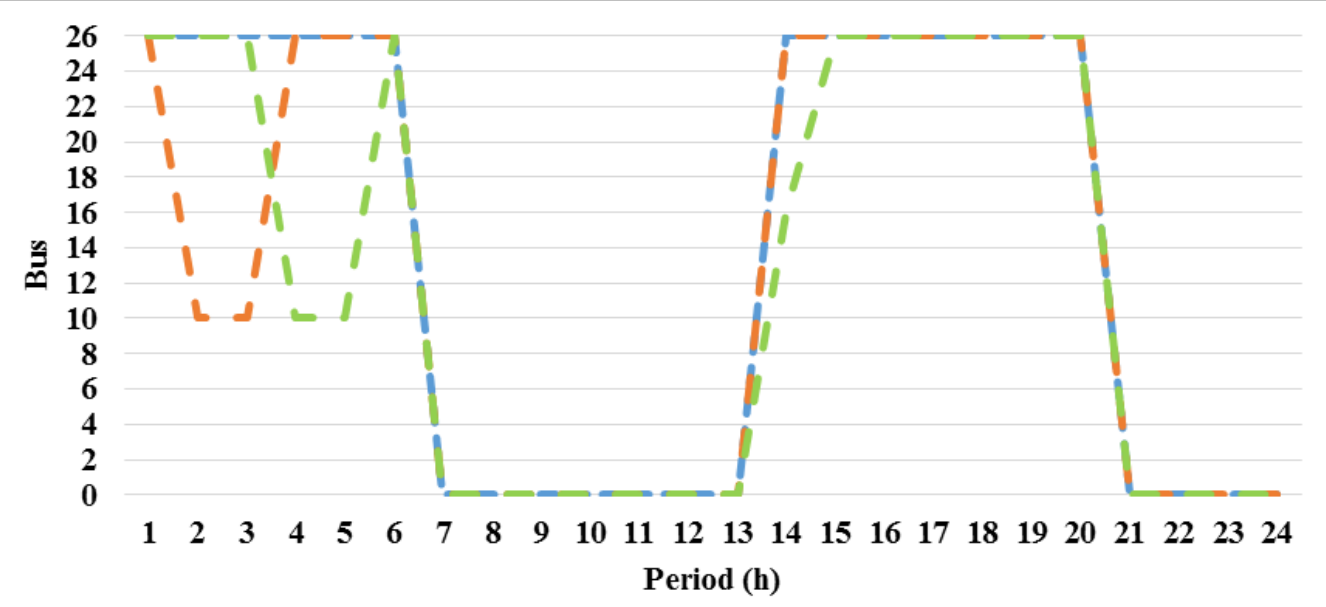

$$
-- \text { State1 }-- \text { State2 }-- \text { State3 }
$$

Fig.10. Top-3 states for the EV-9 


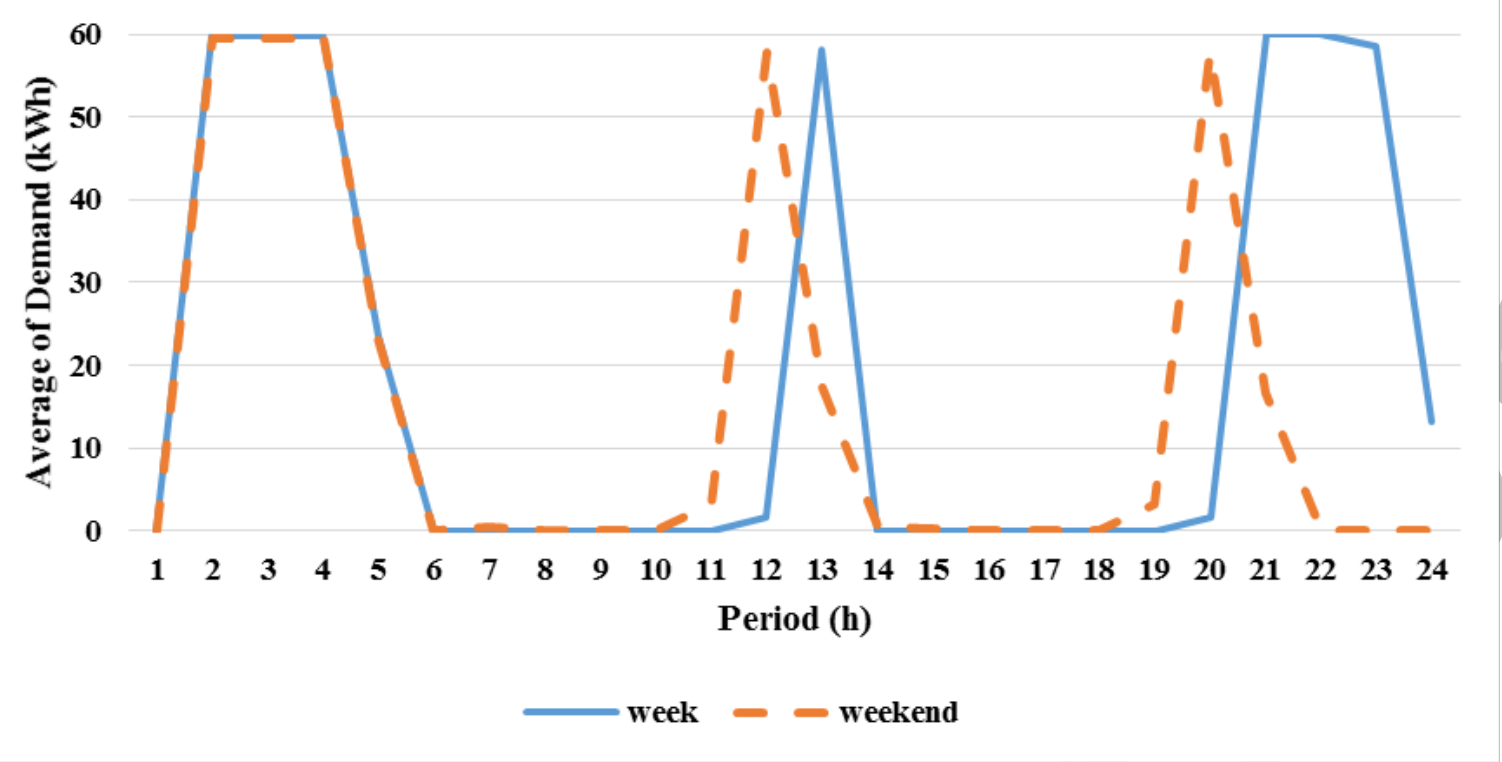

Fig.11. EV-1 average energy demand 


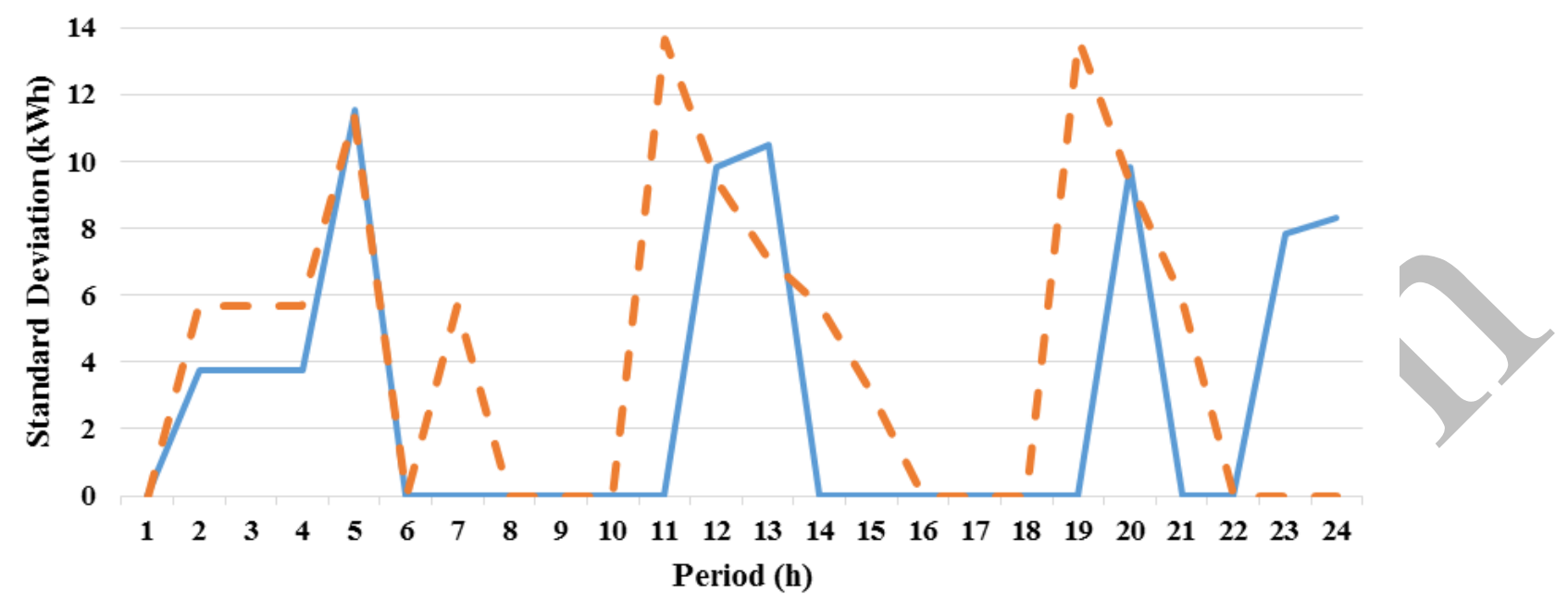

week - - weekend

Fig.12. EV-1 standard deviation of energy demand 


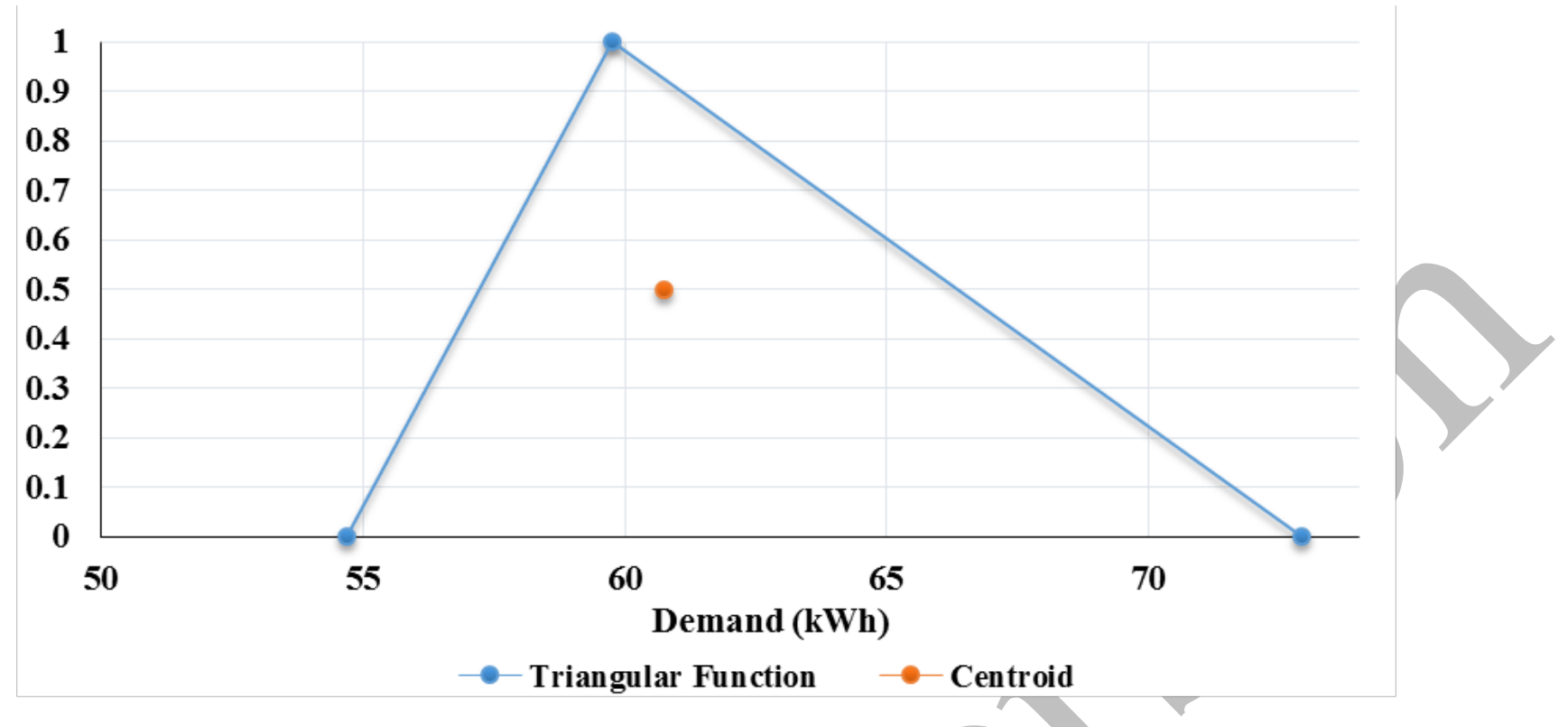

Fig.13. Representation of fuzzy for the average in EV-1 (pessimistic case) 


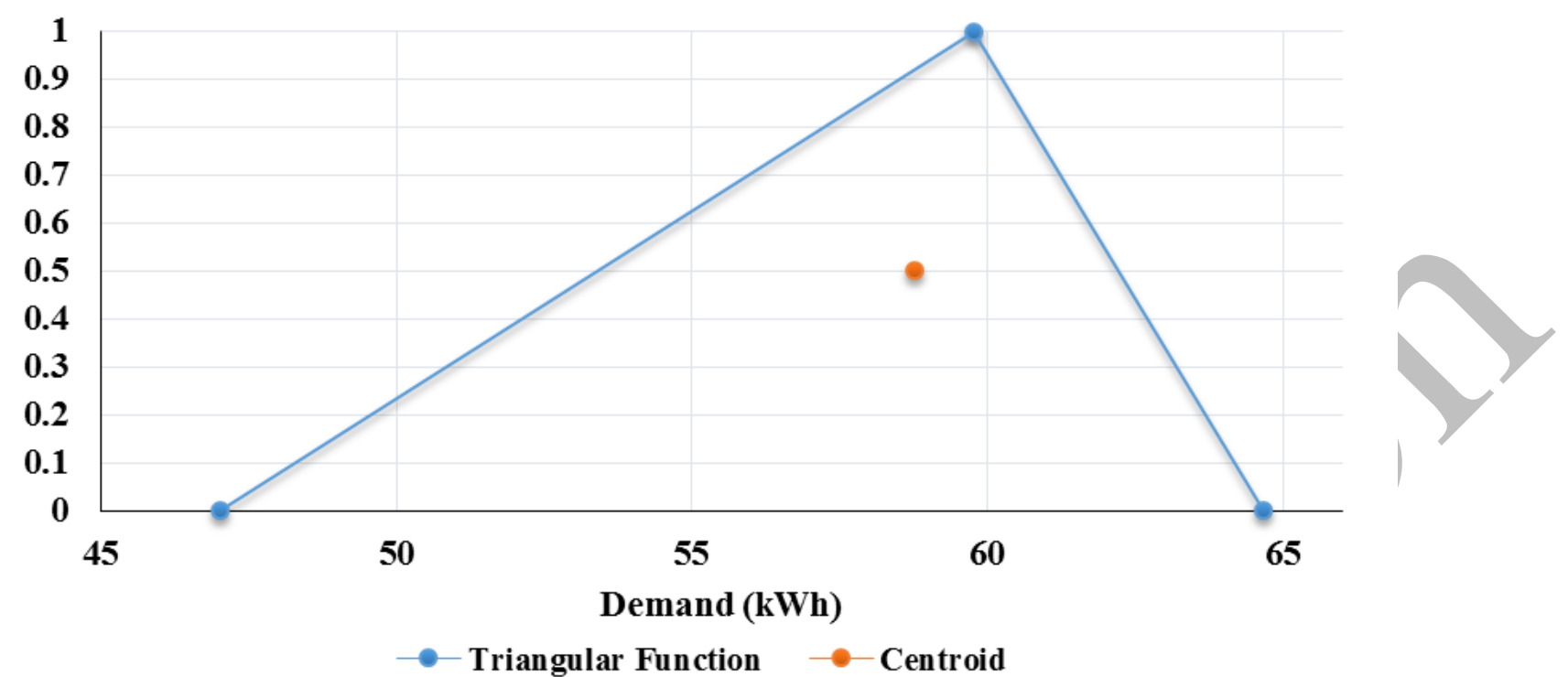

Fig.14. Representation of fuzzy for the average in EV-1 (optimistic case) 


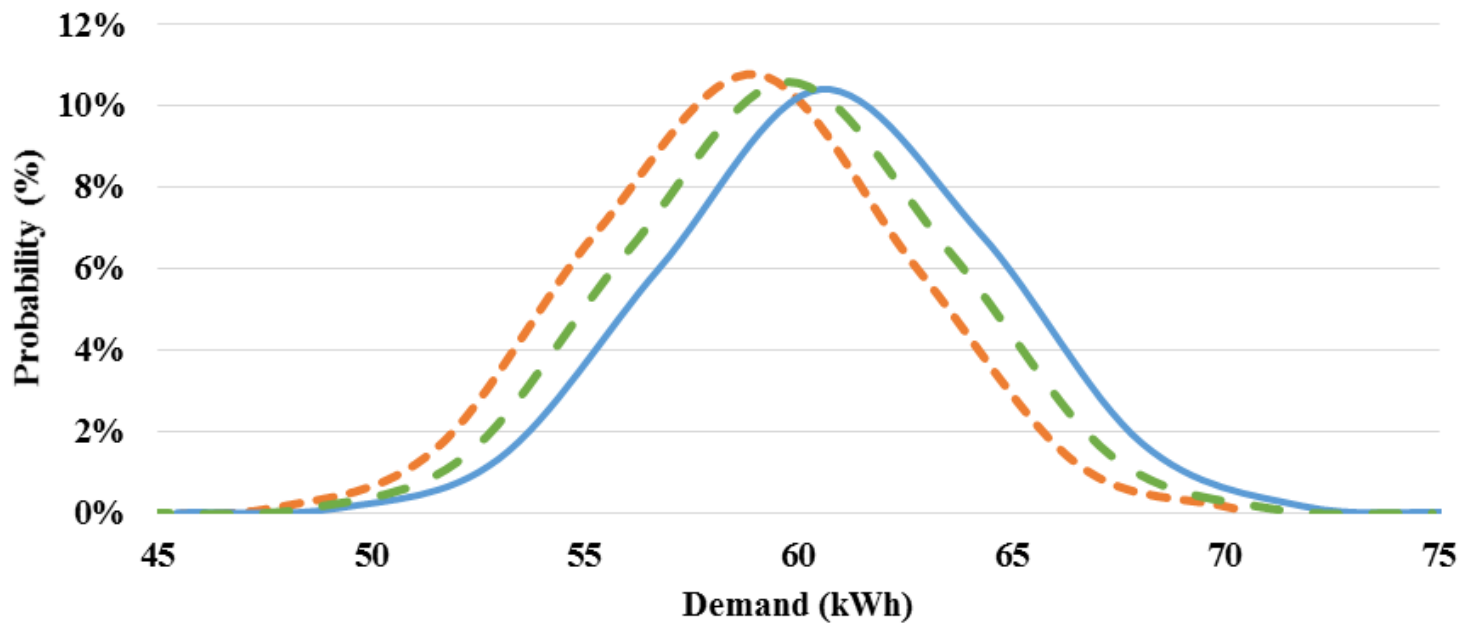

-- Fuzzy_Optimistic $\longrightarrow$ Fuzzy_Pessimistic $\quad-$ Normal Distribution

Fig.15. Probability distribution functions 


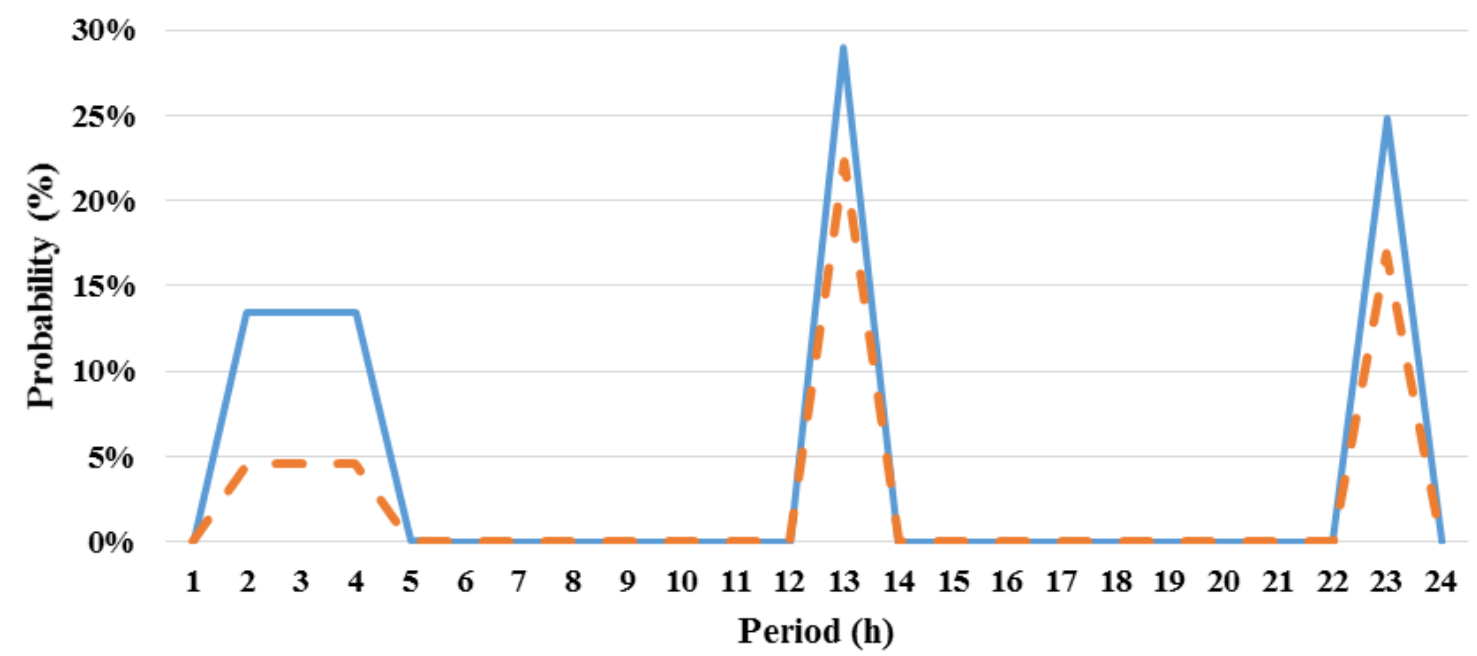

Pessimistic - Optimistic

Fig.16. Probability of energy demand higher than $20 \mathrm{kWh}$ 
Table 1. Groups of EVs (State 1 - probability of 67\%)

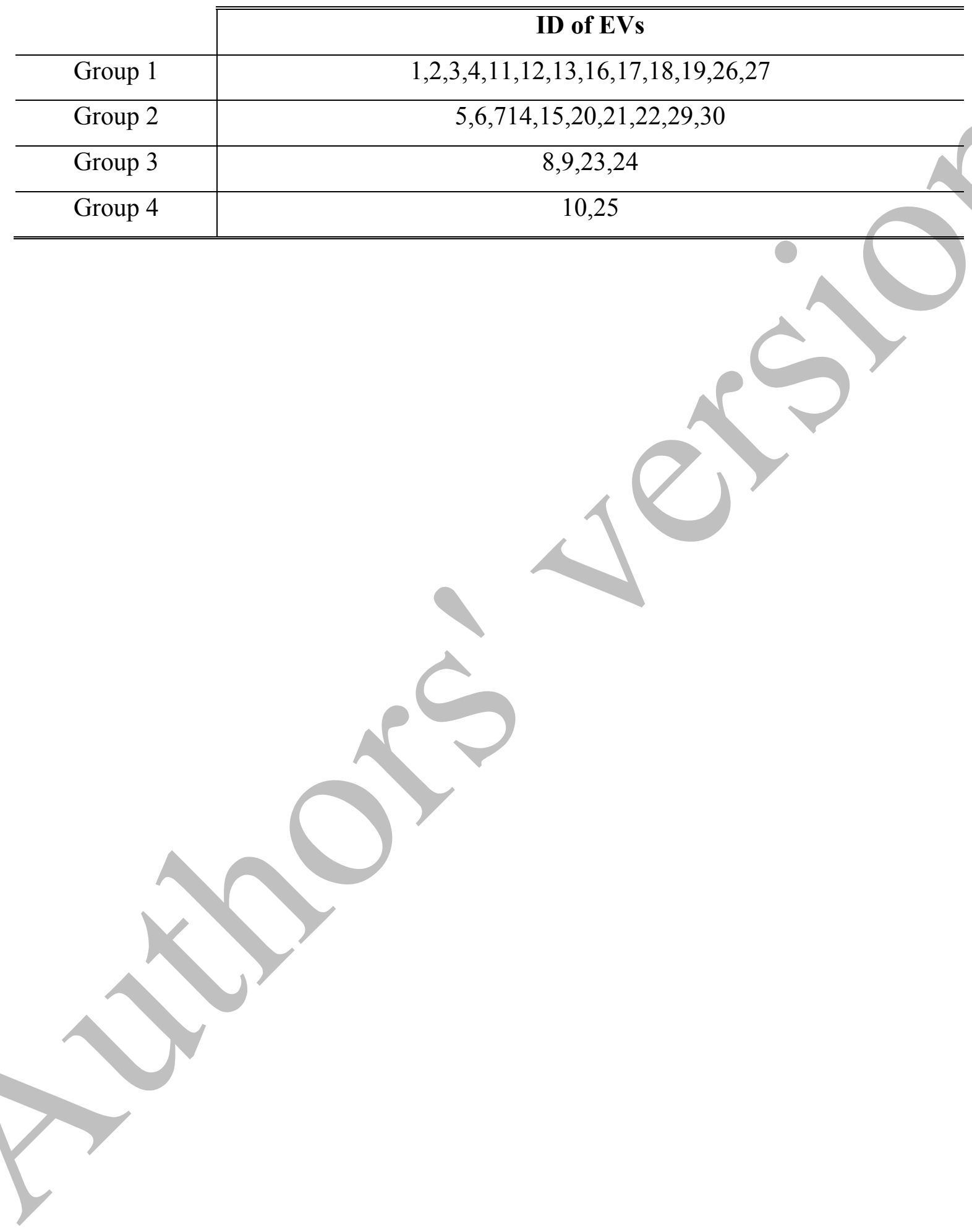


Table 2. State 1 for the 4 groups (bus location)

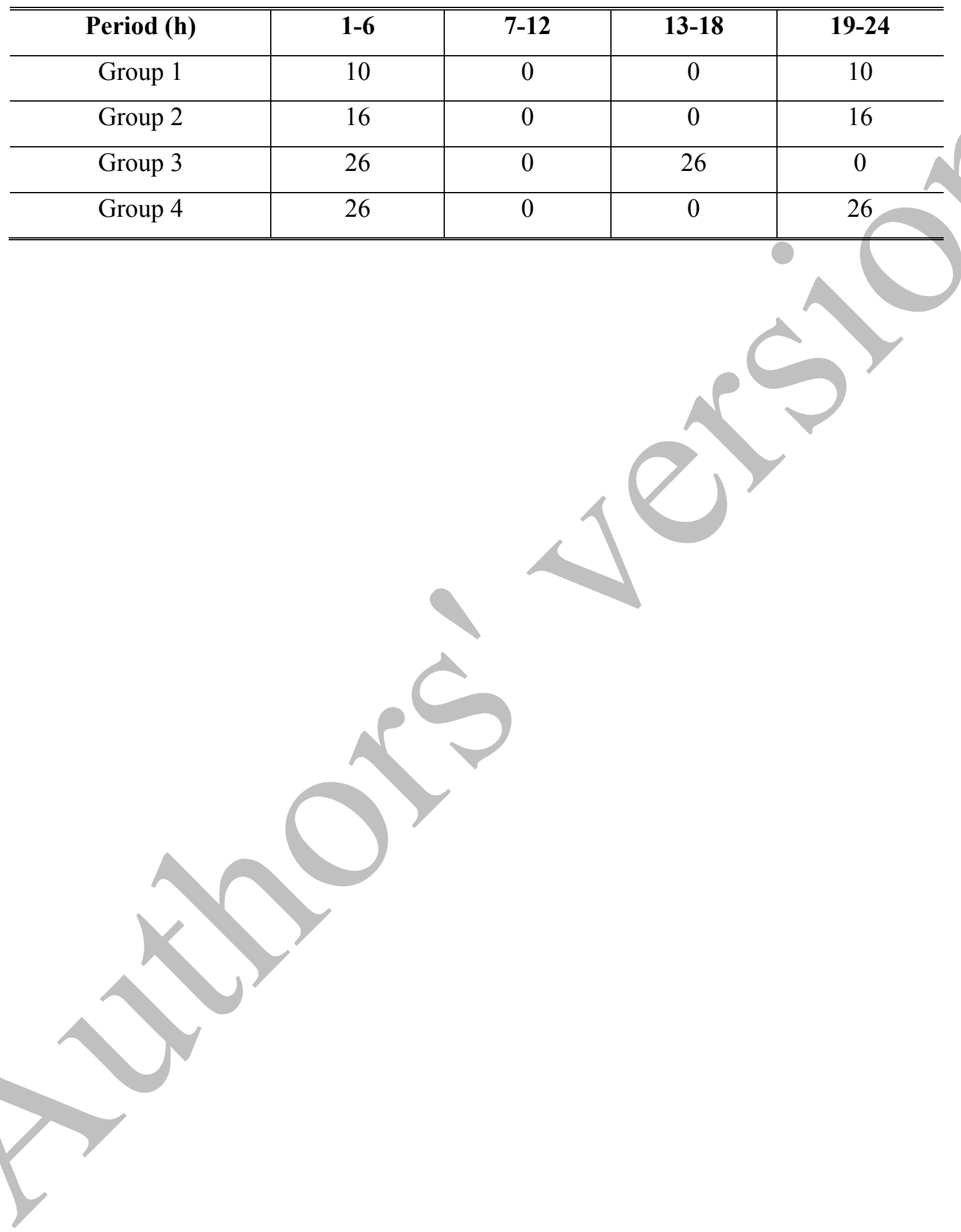


Table 3. Average fuzzy values for EV-1 in the hour $2(\mathrm{kWh})$

\begin{tabular}{c|c|c|c|c}
\hline \hline Case & Average & Lower bound & Fuzzy Value & Upper bound \\
\hline \hline Pessimistic & \multirow{2}{*}{59.76} & 54.68 & 60.76 & 72.91 \\
\cline { 3 - 5 } & & 47.01 & 58.77 & 64.64 \\
\hline \hline
\end{tabular}


Table 4. Standard deviation fuzzy values for EV-1 in the hour $2(\mathrm{kWh})$

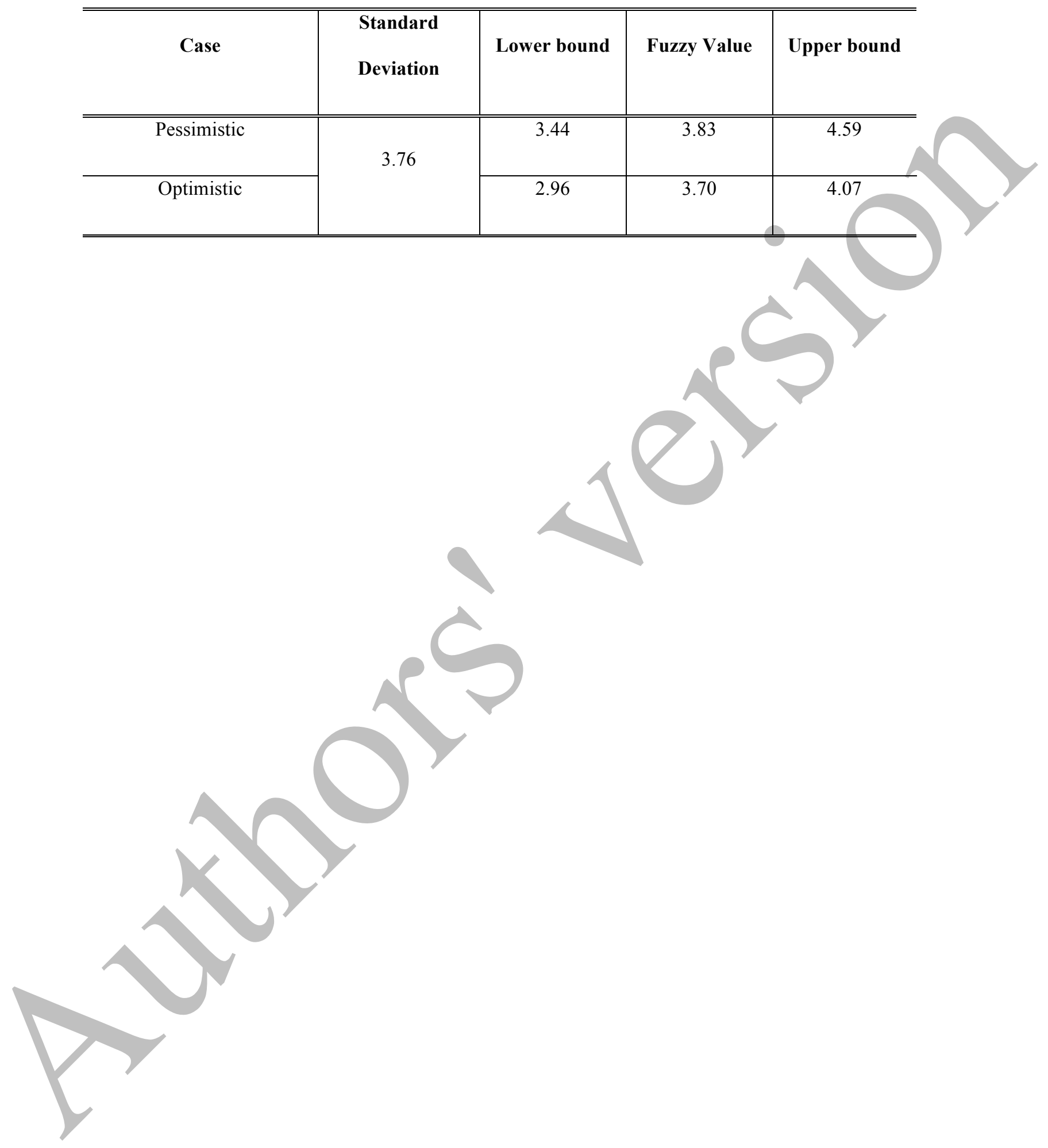

